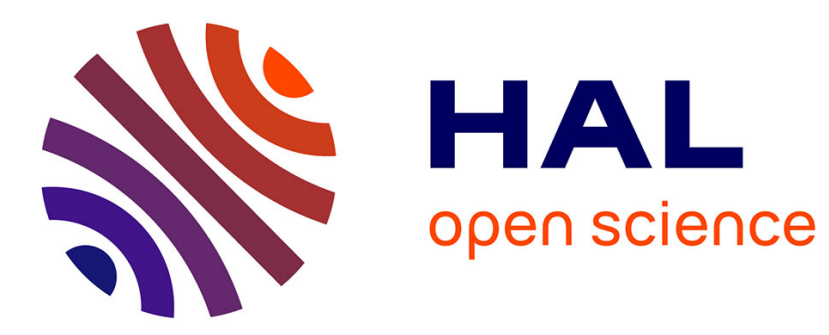

\title{
Transport properties of a Bentheim sandstone under deformation
}

\author{
L. Jasinski, D. Sangare, P. M. Adler, Valeri V. Mourzenko, Jean-François \\ Thovert, N. Gland, Samir Bekri
}

\section{- To cite this version:}

L. Jasinski, D. Sangare, P. M. Adler, Valeri V. Mourzenko, Jean-François Thovert, et al.. Transport properties of a Bentheim sandstone under deformation. Physical Review E: Statistical, Nonlinear, and Soft Matter Physics, 2015, 91 (1), pp.013304(16). 10.1103/PhysRevE.91.013304 . hal-01196392

\section{HAL Id: hal-01196392 \\ https://hal.science/hal-01196392}

Submitted on 29 Jan 2021

HAL is a multi-disciplinary open access archive for the deposit and dissemination of scientific research documents, whether they are published or not. The documents may come from teaching and research institutions in France or abroad, or from public or private research centers.
L'archive ouverte pluridisciplinaire HAL, est destinée au dépôt et à la diffusion de documents scientifiques de niveau recherche, publiés ou non, émanant des établissements d'enseignement et de recherche français ou étrangers, des laboratoires publics ou privés. 


\title{
Transport properties of a Bentheim sandstone under deformation
}

\author{
L. Jasinski* \\ UPMC Sisyphe. Boîte 105, 4 place Jussieu, 75252 Paris Cedex 05, France \\ and Institut Pprime, CNRS, SP2MI, BP 30179, 86962 Futuroscope Cedex, France \\ D. Sangaré ${ }^{\dagger}$ and P. M. Adler ${ }^{\ddagger}$ \\ UPMC Sisyphe. Boîte 105, 4 place Jussieu, 75252 Paris Cedex 05, France \\ V. V. Mourzenko ${ }^{\S}$ and J.-F. Thovert" \\ Institut Pprime, CNRS, SP2MI, BP 30179, 86962 Futuroscope Cedex, France \\ N. Gland ${ }^{\text {II }}$ and S. Békri ${ }^{\#}$ \\ IFP, Institut Français du Pétrole (IFP), 1 et 4, avenue de Bois-Préau, 92852 Rueil-Malmaison Cedex, France
}

(Received 22 May 2014; published 8 January 2015)

\begin{abstract}
The mechanical and transport properties of a Bentheim sandstone are studied both experimentally and numerically. Three classical classes of loads are applied to a sample whose permeability is measured. The elasticity and the Stokes equations are discretized on unstructured tetrahedral meshes which precisely follow the deformations of the sample. Numerical results are presented, discussed, and compared to the available experimental data.
\end{abstract}

DOI: 10.1103/PhysRevE.91.013304

PACS number(s): 47.11.Df, 46.25.-y, 47.56.+r

\section{INTRODUCTION}

The influence of stress on rock properties is important for the oil industry for several applications. First, the decrease of pore pressure during oil production induces significant modifications of the field stress, which results in an increase of the compaction which may have an important impact on the rock microstructures and on their petrophysical properties. Second, plugs which are cut at large depths are analyzed in the laboratory where the pressure is significantly less than in place; this pressure change is likely to have a significant influence on the rock properties.

The major objectives of this paper is to determine the geometrical properties, the conductivity and the permeability of a real sample of Bentheim sandstone whose structure is determined by computed microtomography (CMT). Then this sample is subjected to external stresses and the overall mechanical and transport properties are determined for the deformed sample.

The literature directly related with this subject can be summarized as follows. The authors of Ref. [1] conducted hydrostatic and triaxial compression tests on nominally dry samples of Bentheim sandstone with a porosity of about $23 \%$. The authors of Ref. [2] measured the ultrasonic $P$-wave velocity, the electrical conductivity, and the magnetic susceptibility on Bentheim and Rothbach sandstones. This contribution was completed by a microstructural analysis of these two

\footnotetext{
*lucasz.jasinski@etu.upmc.fr

†sangaredavid@yahoo.fr

†pierre.adler@upmc.fr

§murzenko@ensma.fr

"thovert@ensma.fr

IIngland@slb.com

" samir.bekri@ifpen.fr
}

sandstones [3] which analyzed the anisotropy of elastic and transport properties in the undeformed materials. The authors of Ref. [4] developed a novel apparatus capable of simultaneously measuring permeability, porosity, and ultrasonic velocities at hydrostatic pressures up to $100 \mathrm{MPa}$; they applied it to Bentheim and Crab Orchard sandstones. The authors of Ref. [5] proposed an integrated approach based on an extended rock characterization, an experimental investigation of pressure dependency of directional rock permeabilities, and, finally, a pore scale simulation of this dependency using an equivalent pore network extracted from microtomography analysis; this was conducted on a high-permeability Bentheim sandstone and a dual porosity bioclastic carbonate, the Estaillades limestone having an intermediate permeability. Regarding the Bentheim sandstone, the same tomographic data have been used in Ref. [6] to condition other stochastic reconstruction techniques, by thresholded Gaussian fields or by penetrable spheres models, and to compare the predicted properties to those measured in the CMT image.

The present work is an extension of that in Refs. [5,6], along the same lines as the careful studies conducted by the authors of Refs. [7,8], who computed the conductivity and elastic properties of undeformed Fontainebleau sandstones from tomographic images and compared them to experimental measurements. Here the microstructural characteristics and the macroscopic conduction, flow, and elastic properties are calculated in the undeformed state by solving the local equations in the discretized samples as they are provided by CMT; in addition, the flow and elastic properties are calculated in the deformed state by the same methodology. For these last two quantities, the numerical predictions are compared with experimental measurements performed on the same piece of material. The properties under large deformations are addressed by imposing successive incremental loads. The resulting geometrical changes are accounted for by the progressive deformation of the unstructured tetrahedral mesh, 
which improves on the similar procedure of Ref. [9] for the mechanical response of fractures where the deformations were applied stepwise on a regular mesh.

This paper is organized as follows. The numerical methodology is briefly presented in Sec. II. The measurement procedure, which is fully described by Ref. [5], is summarized in Sec. III. The results are not presented at this stage. The main ones are introduced later simultaneously with the numerical results. Application of microtomography to the Bentheim sandstone is given in Sec. IV where the microstructure is also characterized. The numerical results and their comparison to the experimental data are detailed in Sec. V. First, the conductivity, the permeability, and the mechanical properties of the undeformed samples are determined; second, the samples are deformed along various deformation paths and the last two properties are derived. All the results are compared to the experimental data and thoroughly discussed.

Finally, some remarks and possible extensions are presented in Sec. VI.

\section{GENERAL}

The porous medium is an elastic heterogeneous material whose precise structure is provided by CMT (cf. Sec. IV). The cell $\Omega$ is initially divided into $N_{c}^{3}$ elementary cubes of size $a$ (also called voxels) which are either fully solid or fully void denoted by $\Omega_{s}$ and $\Omega_{p}$, respectively. $\Omega_{p}$ is filled by an incompressible Newtonian fluid of viscosity $\mu$ and electrical conductivity $\Sigma_{o}$. It should be noted that these elementary cells do not remain cubic when they undergo deformation and this necessitates the use of unstructured meshes based on tetrahedra.

This section is devoted to a summary of the determination of the mechanical properties, the macroscopic conductivity, and the permeability of deformed or undeformed materials. We start with the mechanical properties in order to introduce the unstructured meshes.

\section{A. Elastic deformations}

In the solid matrix $\Omega_{s}$, the solid displacement $\mathrm{u}_{s}$ is governed on the microscopic level by the elastic equation

$$
\nabla \cdot \sigma_{s}=0 \text { in } \Omega_{s},
$$

where $\sigma_{s}$ is the stress tensor; the local deformation tensor e can be expressed as

$$
\boldsymbol{e}=\left[\nabla \boldsymbol{u}_{s}+\left(\nabla \boldsymbol{u}_{s}\right)^{t}\right] / 2, \quad \sigma_{s}=\boldsymbol{C}_{\{4\}}: \boldsymbol{e},
$$

where $\boldsymbol{C}_{\{4\}}$ is the solid fourth-order elastic tensor. For isotropic materials, the expression of the stress tensor reduces to

$$
\boldsymbol{\sigma}_{s}=\lambda_{s}\left({ }^{\mathrm{tr}} \boldsymbol{e}\right) \boldsymbol{I}+2 \mu_{s} \boldsymbol{e},
$$

where $\lambda_{s}$ and $\mu_{s}$ are the Lamé coefficients and I the unit tensor. The effective macroscopic stress $\left\langle\sigma_{s}\right\rangle$ is defined as the volume average of the local stress over the total volume.

These equations are discretized and solved as follows. Each elementary cube in the CMT data is discretized by tetrahedra as shown in Fig. 1. Then the elastic equation is integrated over elementary volumes surrounding each corner of each tetrahedron; these volumes are a partition of the solid space

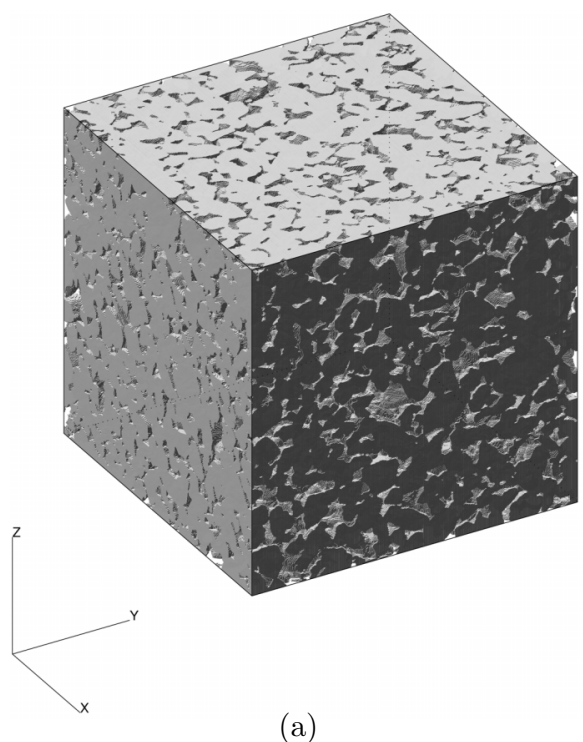

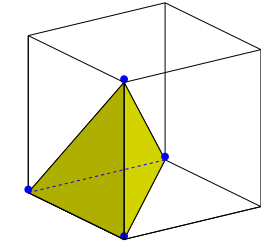

(b)

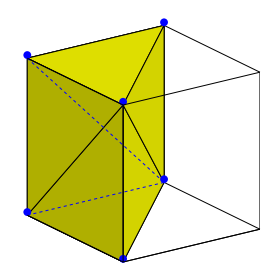

(c)
FIG. 1. (Color online) Discretization of the porous medium. (a) The 3D CMT image of the Bentheim sandstone sample with $500^{3}$ voxels of size $6 \mu \mathrm{m}$. Each voxel is decomposed into six identical tetrahedra in order to obtain the structured mesh $\mathrm{SCT}_{6}$; one tetrahedron is shown in (b) and three of them in (c) where one of the symmetry planes is visible.

$\Omega_{s}$. The resulting linear system is solved by a conjugate gradient technique. Some additional details can be found in Refs. [10,11]. Of course, after deformation, the coordinates of the corners of the elementary tetrahedra are no more integer multiples of the size $a$ of the initial voxels. A check for mesh intersection is made after application of each incremental deformation, and the simulation would stop if it occurred. However, it never does in practice; this would require very large deformation rates (of the order of unity) or discontinuities in the deformation field due to the presence of fractures, for instance. Then the equations are discretized again on the deformed mesh whenever it is needed.

These calculations were performed in three different cases, each of them corresponding to an experiment [5]; they are illustrated in Fig. 2. In the hydrostatic load, equal normal displacements are imposed on the six faces of the cell [see Fig. 2(a)]. In the oedometric load, a constant displacement is imposed on the upper face along the $z$ axis while no normal displacement is allowed on the opposite face nor on the four lateral faces, as illustrated in Fig. 2(b). In the last case called the uniaxial load, a constant displacement is imposed on the upper face along the $z$ axis while the normal displacement on lower face is zero; the four lateral faces are completely free to move. In all cases, the tangential stress is always zero at all the sample boundaries, except in a variant of the uniaxial test, where tangential displacements were also prevented at the lower face.

The various tests give direct access to different macroscopic elastic moduli. The hydrostatic load provides only the value of the bulk modulus,

$$
K_{e}=\frac{{ }^{\operatorname{tr}}\langle\boldsymbol{\sigma}\rangle}{3^{\operatorname{tr}}\langle\boldsymbol{e}\rangle}
$$




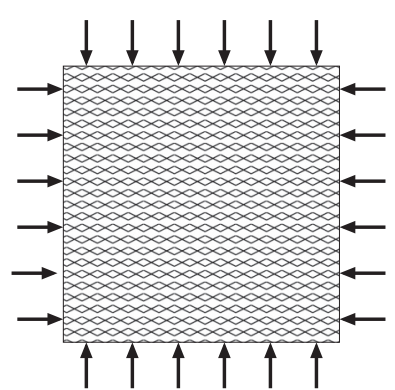

(a)

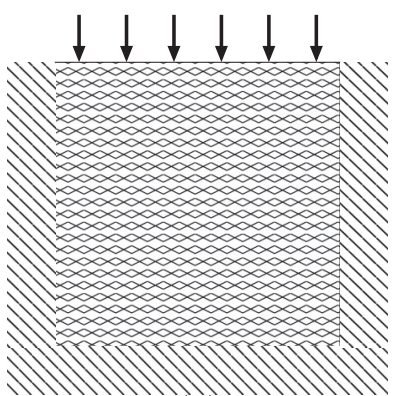

(b)

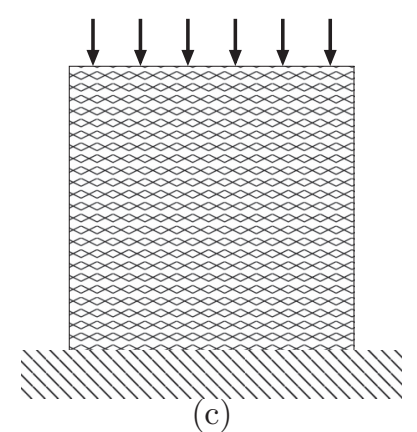

(c)

FIG. 2. Boundary conditions for hydrostatic (a), oedometric (b), and uniaxial (c) loads. Arrows denote imposed normal displacement and zero tangential stress. A shaded casing denotes zero normal displacement and tangential stress. Displacement is unrestricted on the faces without arrows or casing.

The two Lamé coefficients $\lambda_{e}$ and $\mu_{e}$ can be obtained from the oedometric test by

$$
\lambda_{e}=\frac{\left\langle\sigma_{x x}\right\rangle}{\left\langle e_{z z}\right\rangle}, \quad \mu_{e}=\frac{\left\langle\sigma_{z z}\right\rangle-\left\langle\sigma_{x x}\right\rangle}{2\left\langle e_{z z}\right\rangle},
$$

or from similar formula based on $\left\langle\sigma_{y y}\right\rangle$ instead of $\left\langle\sigma_{x x}\right\rangle$. The difference between these two determinations provides a measure of the degree of anisotropy. Finally, the uniaxial test yields the Young modulus and Poisson ratio $E_{e}$ and $v_{e}$,

$$
E_{e}=\frac{\left\langle\sigma_{z z}\right\rangle}{\left\langle e_{z z}\right\rangle}, \quad v_{e}=-\frac{\left\langle e_{x x}\right\rangle}{\left\langle e_{z z}\right\rangle} .
$$

Again, the difference between the values of $v_{e}$ deduced from $\left\langle e_{x x}\right\rangle$ or $\left\langle e_{y y}\right\rangle$ measures the sample anisotropy.

The numerical results are normalized by the corresponding moduli $K_{s}, \lambda_{s}, \mu_{s}$, and $E_{s}$ of the solid material, which has a Poisson ratio $v_{s}=0.07$ representative of quartz. When these results are applied to a real situation with the same value of $v_{s}$, they should be multiplied by the real corresponding moduli as it is done for instance in (24).

\section{B. Conduction}

The electric terminology is used here, but the following developments are also valid for thermal conduction and for diffusion of Brownian particles whose size is small with respect to a typical pore size. The local flux $\boldsymbol{q}$ is equal to

$$
\boldsymbol{q}=-\Sigma_{o} \nabla \psi
$$

where $\Sigma_{o}$ is the fluid conductivity and $\psi$ is the local electrical potential. Electrical and thermal conductions are both governed by a Laplace equation (see Refs. [12] and [13] where additional details are given) which corresponds to the conservation of the local electrical flux,

$$
\nabla^{2} \psi=0,
$$

together with the no-flux boundary condition at the wall $S_{p}$ when the solid phase is assumed to be insulating,

$$
\boldsymbol{n} \cdot \boldsymbol{\nabla} \psi=0 \text { on } S_{p},
$$

where $\boldsymbol{n}$ is the unit vector normal to $S_{p}$.

These equations are solved in cubic blocks of size $a N_{c}$ taken from the CMT image. When considering conduction along a direction $i=x, y$, or $z$, potentials 0 and $\Delta \psi$ are imposed at the two opposite faces normal to $i$, and periodicity is applied along the two transverse directions. The conductivity coefficient $\Sigma_{i i}$ is deduced from the resulting average electrical flux by

$$
\overline{q_{i}}=-\Sigma_{i i} \frac{\Delta \psi}{a N_{c}} .
$$

In the average, for an isotropic random medium, the conductivity tensor $\boldsymbol{\Sigma}$ is spherical and equal to $\boldsymbol{\Sigma} \boldsymbol{I}$. The formation factor $F$ is generally defined as the inverse of the dimensionless macroscopic conductivity,

$$
F=\frac{\Sigma_{o}}{\Sigma}
$$

The Neumann problem [(4) and (5)] is solved via a secondorder finite-difference formulation. The resulting set of linear equations is solved by a conjugate gradient algorithm.

Several boundary conditions combining Dirichlet and Neumann conditions, periodicity, and insertion of empty layers have been tested and found to yield nearly equal results for sufficiently large block sizes, including those considered here. Only the initial configurations were studied since there is no experimental data to compare with. Full details are provided in Ref. [14].

\section{Stokes flow}

The low-Reynolds-number flow of an incompressible Newtonian fluid is governed by the Stokes equations,

$$
\nabla p=\mu_{f} \nabla^{2} v, \quad \nabla \cdot v=0,
$$

where $\boldsymbol{v}, p$, and $\mu_{f}$ are the velocity, pressure, and viscosity of the fluid. In general, $v$ satisfies the no-slip condition at the solid walls,

$$
\boldsymbol{v}=0 \quad \text { on } \quad S_{p} .
$$

For each direction of calculation, the cubic blocks are supplemented with an empty layer normal to the mean flow direction, and periodic boundary conditions are applied. A macroscopic pressure gradient $\overline{\nabla p}$ induces a flow characterized by the seepage velocity $\bar{v}$ which obeys the Darcy law,

$$
\overline{\boldsymbol{v}}=-\frac{1}{\mu_{f}} \boldsymbol{K} \cdot \overline{\nabla p} .
$$


Here $\boldsymbol{K}$ is a symmetric tensor that is positive definite. It only depends on the geometry of the system and thus can be simplified when the porous medium possesses geometric symmetries. Its diagonal component $K_{x x}$ was calculated by imposing $\overline{\nabla p}$ along the $x$ axis. It is simply denoted by $K$ in the following.

Two kinds of boundary conditions have been tested, with either an impermeable casing or periodic conditions in the directions transverse to the flow. Along the flow direction, periodic boundary conditions are applied in both cases to the cubic block supplemented with a free fluid layer. The two configurations are found to yield nearly equal results for sufficient large block sizes [14]. Note that the disturbing effects of the transverse conditions are of a different nature and of a much milder amplitude than those quantified by Ref. [15] in permeameter experiments, which resulted from fluid bypassing around the core and/or rock damage induced by the coring procedure.

Two numerical solvers of the Stokes equations have been used which are based on finite volume formulations applied to unstructured or cubic meshes $[11,16,17]$ depending on the deformed or undeformed character of the medium. In order to cope with the continuity equation, the so-called artificial compressibility method was applied [18]. The solver which operates on a regular cubic grid is much faster and therefore it was used for the exhaustive calculations in the very large number of sub-blocks with size up to $250^{3}$ investigated in the undeformed state. However, since only the solver based on an unstructured mesh can handle the successive deformations induced by the incremental loads, it was used for the investigation of the flow properties under load. For this reason, and also because the flow calculations have to be repeated in sequences of successive deformed states, fewer and smaller samples have been considered.

\section{EXPERIMENTAL MEASUREMENTS}

The triaxial cell used in this study has been codeveloped by I.F.P. and ErgoTech. The cell is operated using two highpressure pumps to control independently the confining and axial pressures in the range 3 to $69 \mathrm{MPa}$. An intermediate pressure pump generates flows of brine through the core sample. The pore pressure regulation, up to $25 \mathrm{MPa}$, is achieved either by a back-pressure system or by the pump. The cell is placed inside an oven where the temperature can be regulated up to $200{ }^{\circ} \mathrm{C}$. Care is taken with thermal regulation of the whole system, critical for accurate measurements of permeabilities on long periods. All results presented in this study were obtained at a regulated temperature of $40^{\circ} \mathrm{C}$. The cell is equipped with deformation sensors to measure both axial and radial strains of the core samples. The displacement of the upper mobile piston is monitored using a couple of external linear variable differential transformer (LVDT) sensors fastened parallel between the two pistons. The radial deformation of the core is measured using a double cantilever sensor fastened to the sleeve inside the cell.

The innovative characteristic of the cell is related to the special equipment of the core sleeve which allows permeabilities measurements in three orthogonal directions. Commonly, permeability measurement in a triaxial setup is achieved by

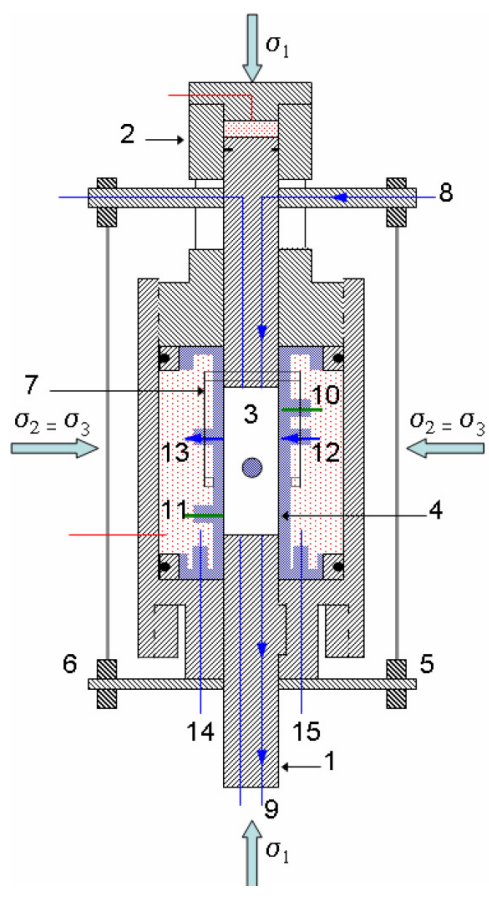

$\begin{array}{ll}\text { 1. } & \text { Fixed piston } \\ 2 . & \text { Mobile piston } \\ 3 . & \text { Sample } \\ \text { 4. } & \text { Core sleeve } \\ 5,6 . & \text { RDP LVDT } \\ 7 . & \text { Internal cantilever } \\ 8,9 . & \text { Axial flow lines } \\ 10,11 . & \text { Fluid pressure } \\ & \text { samplers } \\ 12,13 . & \text { Radial injectors } \\ 14,15 . & \text { External tubing }\end{array}$

FIG. 3. (Color online) Simplified sketch of the directional flow triaxial cell.

measuring during steady-state flow the differential of pore pressure between the inlet and the outlet of the sample along the axial direction, which is also the direction of maximal stress. In addition, our triaxial setup allows us to measure an intermediate pressure difference (between two points located at $1 \mathrm{~cm}$ of the core ends) using small fluid samplers in contact with the lateral surface of the core (items 10 and 11 in Fig. 3), in order to suppress the possible end effects on axial permeability measurement [19]. The first permeability measurement $K_{a \text {,FL }}$ is referenced as the axial full-length, while the second one, $K_{a, \mathrm{ML}}$, is referenced as the axial midlength. The core sleeve is also equipped to measure the permeabilities $K_{r}$ in two radial directions along the diameter of the sample. Specific flow ports are positioned in contact with the lateral surface of the core. For each radial direction, there is one injector (item 12) facing one receptor (item 13) along the diameter. For the pressure difference measurement, we use a set of differential pressure sensors ranging from \pm 15 to \pm 400 mbar adapted to measure permeabilities of cores of $1 \mathrm{D}$ to $1 \mathrm{mD}$, respectively.

Compression tests are performed vertically on cylindrical samples of diameter $38.1 \mathrm{~mm}$ and height $80 \mathrm{~mm}$ saturated with $\mathrm{NaCl}$ brine at concentration $20 \mathrm{~g} \mathrm{~L}^{-1}$. First, we apply a preliminary hydrostatic pressure of few MPa to ensure sealing of the core sleeve onto the sample and the pore fluid pressure is increased to $1 \mathrm{MPa}$ using a back-pressure system. At this point, the oven as well as the pore fluid pump and the flow lines network are regulated at $40{ }^{\circ} \mathrm{C}$. At this prestressed and equilibrated stage, all displacement sensors are initialized to zero. Two different stress paths were investigated during our compression tests; for hydrostatic compression experiments, we performed the loading by pressure steps towards the maximum pressure at a constant pore pressure of $1 \mathrm{MPa}$; for the "uniaxial" compression tests, the increase of axial stress 
was continuous and the confining pressure was maintained at $3 \mathrm{MPa}$. At each pressure step, we performed axial and radial permeability measurements using the steady-state method. For the two axial measurements, the classical Darcy law (10) is used. For the two radial measurements, a modified Darcy law was employed with a correction factor introduced to take into account the noncylindrical shape of the radial flow lines between the transverse flow ports of cross section along the sample diameter $D$. Details about this correction factor and its estimation in the present setup are given in Ref. [5].

\section{MICROTOMOGRAPHY AND MICROSTRUCTURAL CHARACTERIZATION}

\section{A. Microtomography}

The CMT equipment installed at IFP is a Nanotom from PHOENIX X-Ray (Germany). The source is generated by the impact of a focused electron beam on a thin target. Spot size varies between 1 and $5 \mu$ depending on the operating conditions. The diverging geometry of the X-ray source results in a magnification of the object image. The detector is a Hamamatsu flat detector $(110 \mathrm{~mm} \times 110 \mathrm{~mm})$ made of $2304 \times 2304$ pixels, $50 \mu$ in size. Generating images using microfocus computed tomography (CT) starts with the acquisition of a series of $2 \mathrm{D}$ projections while progressively rotating the sample step by step through a full $360^{\circ}$ rotation at increments of less than $1^{\circ}$. The entire diameter of the sample should remain within the field of view throughout the entire $360^{\circ}$ rotation; a 5-mm-diameter sample is completely displayed on each projection with a pixel size of $3 \mu$.

Acquisition parameters for rock analysis were the following: 5-mm-diameter sample; source object distance, $12 \mathrm{~mm}$; source-detector distance, $200 \mathrm{~mm}$; pixel size, $3 \mu$; $2000 \times 2000$ field of view, $90 \mathrm{KV} ; 170 \mu \mathrm{A} ; 0.2^{\circ}$ angular step; $\mathrm{Cu}$ 0.1-mm filter; and 2.5-h acquisition time. Each acquisition generates 1800 TIFF projections coded in 12 bits (about $10 \mathrm{Mo}$ each), which represents about 18 Go per data set. These data sets are used for the numerical reconstruction of the volumetric data.

The reconstruction (PHOENIX algorithm) uses a classical cone-beam Feldkamp algorithm. CMT images are corrected from beam hardening effect both by using a metal filter $(\mathrm{Cu})$ and by using a mathematical correction during the reconstruction process (Phoenix). Because of the relatively low resolution, it was not found necessary to correct for ring artifacts.

Reconstructions were performed in 16 bits on a fourPC cluster of 64-bit dual-core CPUs. The maximum unit volume which can be reconstructed at full resolution is $1000 \times 1000 \times 500$ (about a 25 -min reconstruction). For each sample, two consecutive high-resolution volumes $(1000 \times 1000 \times 500)$ are reconstructed and appended to form the basic $1000 \times 1000 \times 1000$ volume on which the analysis is done. Then subset volumes can be extracted from this $1000 \times 1000 \times 1000$ basic volume. In order to reduce their disk size and allow a larger volume access, the volumes can be converted into a 8-bit format or/and binned if the full resolution is not needed.
A single cubic sample of Bentheim sandstone was reconstructed by means of CMT with two different voxel sizes $a$ equal to 3 and $6 \mu \mathrm{m}$. The two resolutions correspond to cubic arrays of $1000^{3}$ and $500^{3}$ voxels, respectively. The second case is illustrated in Fig. 1(a).

The microstructural properties and the transport properties of the initial configurations have been measured or calculated in both cases and the results were found to differ only slightly. For instance, porosity is found equal to 0.232 and 0.240 with the coarse and fine resolutions, respectively. Macroscopic conductivity and permeability of the initial configurations are found to be larger by a few percentages when computed from the $(1000 \times 3 \mu \mathrm{m})^{3}$ image. A full comparison is provided in Ref. [14].

Precision depends on the resolution and on the overall size of the sample. The influence of resolution is obvious, while statistical fluctuations decrease with the size of the sample. In order to have configurations which could be computed in a reasonable amount of time, a compromise had to be found between these two parameters. It was decided to maximize the volume of the computation domains; the coarse image for the calculations of the elastic response and of the transport properties under load were used. Therefore, all the results discussed in the following pertain to the $(500 \times 6 \mu \mathrm{m})^{3}$ image.

\section{B. Microstructural characterization}

The first important property which can be studied is porosity, which is defined as the volume fraction of the interstitial space,

$$
\epsilon=\frac{\Omega_{p}}{\Omega},
$$

where $\Omega_{p}$ is the volume of the pore space and $\Omega$ is the total volume of the sample under consideration.

The overall sample porosity is equal to 0.232 , which is slightly smaller than the measured value 0.244 in the largerscale sample used in Ref. [5] for the mechanical tests. The porosity profiles along the $x, y$, and $z$ axes are displayed in Fig. 4. These profiles are seen to be stable, i.e., without any systematic trend to increase or to decrease.

The CMT image was sliced into smaller cubic blocks of size $N_{c}^{3}$ voxels with

$$
N_{c}=1,2,4,5,10,20,25,50,100,125,250 .
$$

The porosity $\epsilon\left(N_{c}, i\right)$ was systematically calculated for all the blocks $i$ of size $N_{c}$. Of course, the average value $\left\langle\epsilon\left(N_{c}\right)\right\rangle$ is independent of $N_{c}$, but it is interesting to look at the variations of its standard deviation $\sigma_{\epsilon}\left(N_{c}\right)$ defined by

$$
\sigma_{\epsilon}\left(N_{c}\right)=\left\{\frac{1}{\mathcal{N}} \sum_{i=1}^{\mathcal{N}}\left[\epsilon\left(N_{c}, i\right)-\left\langle\epsilon\left(N_{c}\right)\right\rangle\right]^{2}\right\}^{1 / 2},
$$

where $\mathcal{N}$ is the number of sub-blocks of size $N_{c}$. Results are displayed in Fig. 5 (see also Refs. [20] and [21]). $\sigma_{\epsilon}^{2}$ tends to $\epsilon(1-\epsilon)$ as expected for blocks smaller than the correlation length, of the order of $40 \mu \mathrm{m}$ [see Eq. (17)], since under this condition the blocks sample entirely void or solid regions. The standard deviation decreases for larger block sizes and approaches the usual dependence on the square root of the 


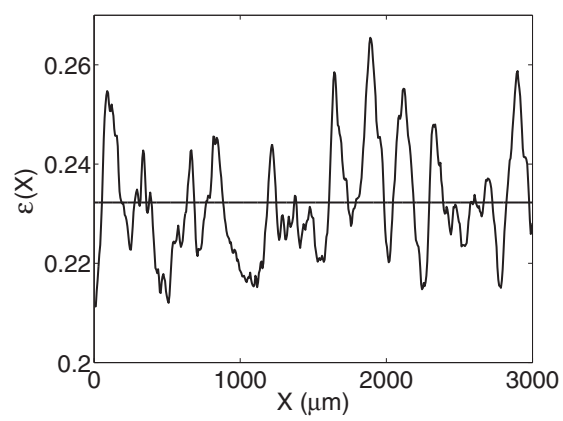

(a)

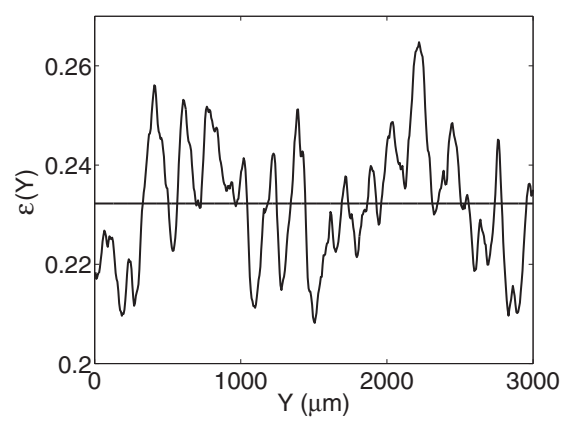

(b)

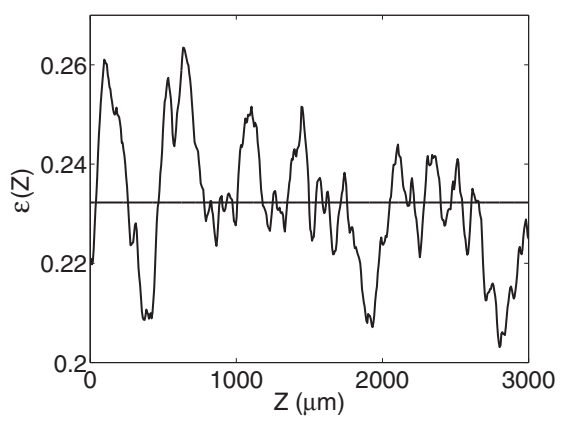

(c)

FIG. 4. Porosity profiles along the $x, y$, and $z$ directions. The straight lines are the averages over the whole block.

sample size $\left(N_{c} a\right)^{3}$ as a consequence of the law of large numbers [22],

$$
\sigma_{\epsilon}\left(N_{c}\right) \propto\left(N_{c} a\right)^{-3 / 2}, \quad\left(300 \mu \mathrm{m} \leqslant N_{c} a \leqslant 750 \mu \mathrm{m}\right) .
$$

The number of blocks becomes too small for larger sizes to assess whether the decay of $\sigma_{\epsilon}$ slows down, as would occur if large-scale porosity fluctuations existed.

Histograms of porosity are systematically displayed in Fig. 6. They are compared to the Gaussian or log-normal distributions deduced from the measured first and second moments. Both laws provide a reasonable approximation of the data set.

The correlation function $R_{Z}(\mathbf{u})$ of the phase function $Z(\mathbf{x})$ is generally defined as [13]

$$
R_{Z}(\mathbf{u})=\frac{\overline{(Z(\mathbf{x})-\epsilon)[Z(\mathbf{x}+\mathbf{u})-\epsilon]}}{\overline{[Z(\mathbf{x})-\epsilon]^{2}}},
$$

where $Z(\mathbf{x})$ is equal to 1 if the point $\mathbf{x}$ belongs to the pore space and 0 otherwise. $\mathbf{u}$ is the lag; the overbar denotes the spatial average. When the medium is isotropic, $R_{Z}(\mathbf{u})$ only depends on the modulus $u$ of $\mathbf{u}$. Correlation lengths $\mathcal{L}_{\alpha}(\alpha=x, y, z)$ can be defined by

$$
\mathcal{L}_{\alpha}=\int_{0}^{\infty} R_{Z}\left(u_{\alpha}\right) d u_{\alpha}
$$

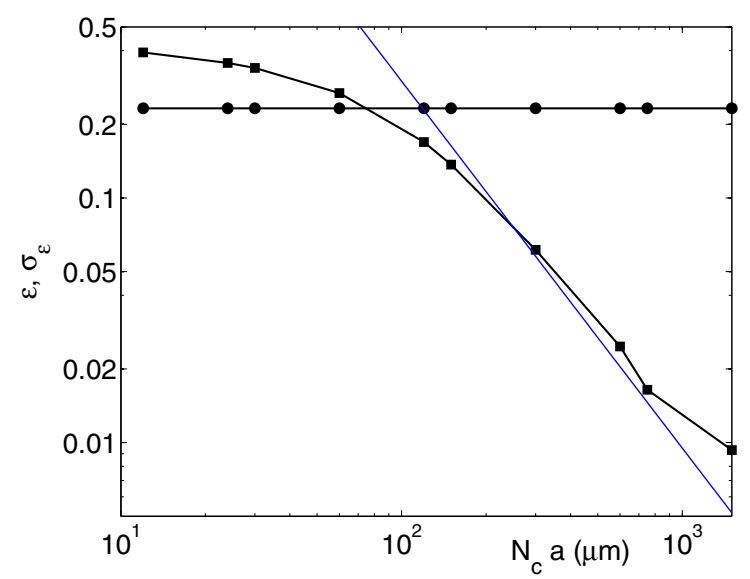

FIG. 5. (Color online) The porosity $\epsilon$ (०) and the standard deviation $\sigma_{\epsilon}\left(N_{c}\right)(\square)$ as functions of the block size $N_{c} a$ (in $\mu \mathrm{m}$ ). The thin straight line is an indication of the slope $-3 / 2$ of the power law (14). where $u_{\alpha}$ is the $\alpha$ component of $\boldsymbol{u}(\alpha=x, y, z)$. Calculations were performed along the $x, y$, and $z$ axes for the whole $500^{3}$ block. Results are given in Fig. 7. The correlation lengths expressed in $\mu \mathrm{m}$ are equal to

$$
\mathcal{L}_{x}=34.7, \quad \mathcal{L}_{y}=38.5, \quad \mathcal{L}_{z}=36.5, \quad \mathcal{L}_{m}=36.6,
$$

where $\mathcal{L}_{m}$ is the average of the three values. A slight anisotropy can be observed with a longer range correlation along the $y$ axis. This fact will be confirmed in Sec. V by the anisotropy of the transport properties.

The hydraulic radius $R_{H}$ is defined as the ratio between the pore volume $\Omega_{p}$ and the surface $S$ of the interface inside the same total volume. Therefore, $R_{H}$ is the inverse of the specific surface. When the medium is discretized by cubic voxels, the surface is overestimated by a factor $3 / 2$. After correction by this factor, $R_{H}$ is found to be equal to $16.5 \mu \mathrm{m}$.

The skeleton of the pore space can be viewed as a simplified image of the pore space, analogous to a capillary network. It is of interest to note that the literature devoted to pore network models is immense; a recent comparison between this type of model and the present one was done in Ref. [23]. The skeleton is determined by a progressive conditional thinning algorithm [24]. It results in a graph which contains $N_{e}$ edges and $N_{v}$ vertices. Various statistical quantities can be measured on the skeleton. Let $d_{s}$ denote the distance of a point on the skeleton to the closest solid. Its minimum $r_{e}$ along the edge $e$ is the critical radius of this edge, i.e., the radius of the largest sphere that can travel along $e . d_{e}$ is the distance between the end points of the edge $e$ and $r_{v}$ the vertex radius, i.e., the value of $d_{s}$ at the vertex location. $z_{v}$ is the coordination number of the vertex $v$, i.e., the number of edges incident on $v$.

The following calculations were performed. In order to illustrate the structure of the sandstone, a $125^{3}$ sub-block cut from the $500^{3}$ image and its skeleton are shown in Fig. 8; they do not present any specific features when compared, for instance, to the ones given in Ref. [20]. Then a systematic analysis was performed in a block of size $N_{c}=250$ cut from the $500^{3}$ image and in eight nonoverlapping sub-blocks of size $123^{3}$. The corresponding histograms are given in Fig. 9. It can be noted that the average results for the sub-blocks of size $123^{3}$ are very close to the ones for the block of size $N_{c}=250$.

When compared to the corresponding results for a lowporosity Fontainebleau sandstone (Fig. 12 in Ref. [20]), the main differences are in the spectra of $r_{e}, r_{v}$, and $d_{s}$. The decrease of the distribution of $r_{e}$ in the Fontainebleau 


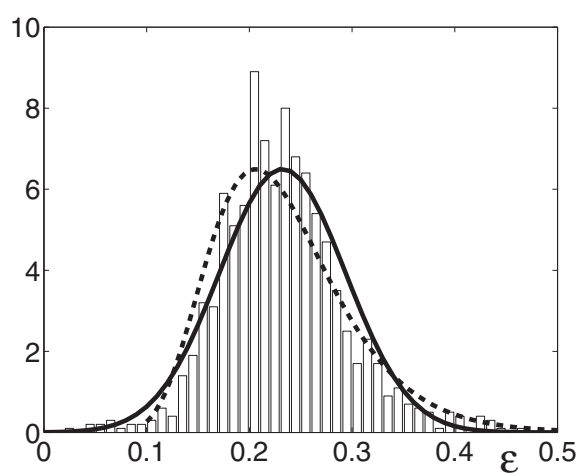

(a)

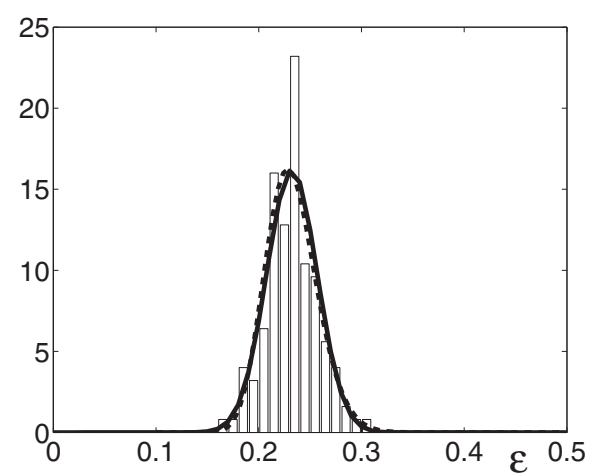

(b)

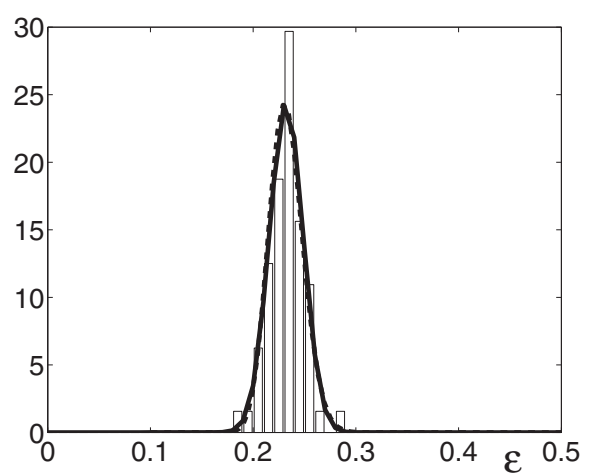

(c)

FIG. 6. Normalized histograms of the porosity $\epsilon$, in blocks of size $N_{c} a=300$ (a), 600 (b), of $750 \mu \mathrm{m}$ (c). The solid and dash curves are the Gaussian and log-normal distributions deduced from the measured averages and standard deviations in real and log-spaces.

sandstone was much steeper than here, and the distributions of $r_{v}$ and $d_{s}$ were monotonously decreasing, while they both have a maximum at about $15-20 \mu \mathrm{m}$ in the Bentheim sandstone. This denotes a larger proportion of bulky cavities in the present sample, resulting in part from its larger porosity ( 0.23 versus 0.069). However, the differences are also due to the better spatial resolution of the present $\mathrm{CT}$ image, relative to the typical pore size quantified by the correlation length, with a ratio of about 6 to 40 here versus only 6 to 25 in Ref. [20].

Finally, percolation was systematically studied in subblocks of increasing sizes 150, 300, 600, 750, and 1500 $\mu \mathrm{m}$, cut from the $(500 \times 6 \mu \mathrm{m})^{3}$ image. All the sub-blocks resulting from the partition of the full image were examined, i.e., $8000,1000,125,64$, or 8 sub-blocks, depending on their size. Percolation was tested between two opposite faces of the sample, with or without transverse periodicity. In all cases, a unit percolation probability is obtained for blocks of $600 \mu \mathrm{m}$ or more, and it is more than 0.99 for blocks of $300 \mu \mathrm{m}$. Significantly smaller values $(\approx 0.75)$ are obtained only for blocks of $150 \mu \mathrm{m}$, i.e., about 4 times $\mathcal{L}_{m}$. This is a striking difference with the corresponding results for the low-porosity Fontainebleau sandstone of Ref. [20]. Blocks of size $4 \mathcal{L}_{m}$ percolated with a probability of only 0.2 , and even with a block size $16 \mathcal{L}_{m}, P_{p}$ was only about 0.6 . This is because the Fontainebleau sandstone was very close to the percolation threshold, due to its low porosity 0.069 . Therefore, size-effects were very important. The present Bentheim sandstone with

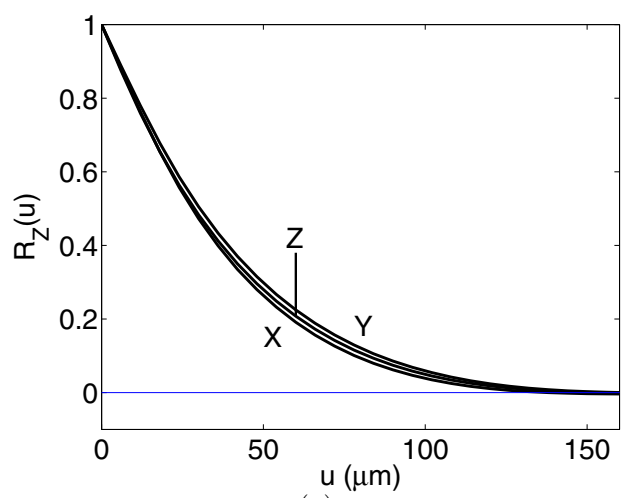

(a) $\epsilon=0.23$ is very far from critical, and any sample reasonably larger than $\mathcal{L}_{m}$ percolates almost certainly.

\section{TRANSPORT AND ELASTIC PROPERTIES}

The transport properties are characterized first of the initial configurations for conductivity and permeability. Then the elastic response of the material is studied for various kinds of loads. Finally, permeability in deformed states is considered.

\section{A. Conductivity of the initial configurations}

Conductivity was only studied in the undeformed samples since there is no experimental data to compare with, but a very complete set of results was obtained. The full conductivity tensor was calculated for various boundary conditions. It was systematically determined for the 125 sub-blocks of size $100^{3}$, the 64 sub-blocks of size $125^{3}$, and the 8 sub-blocks of size $250^{3}$. Overall correlations could be derived as functions of porosity.

Systematic data related to conductivity are represented in two different ways. The first way is to gather all the results obtained for the various directions as functions of the block sizes as done in Fig. 10(a). Two observations can be made about this figure. First, conductivity along the $y$ axis is systematically larger than the two other ones. This corresponds to a similar observation for the percolation length

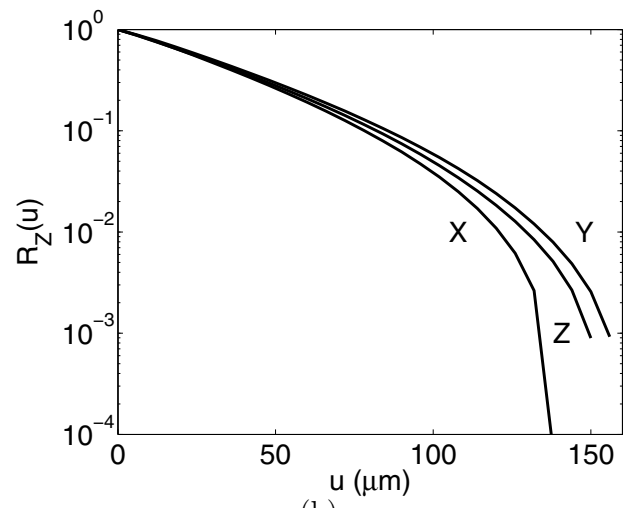

(b)

FIG. 7. (Color online) Correlation functions $R_{Z}$ as a function of the lag $u$ in $\mu \mathrm{m}$ along the $x, y$, and $z$ axes. 

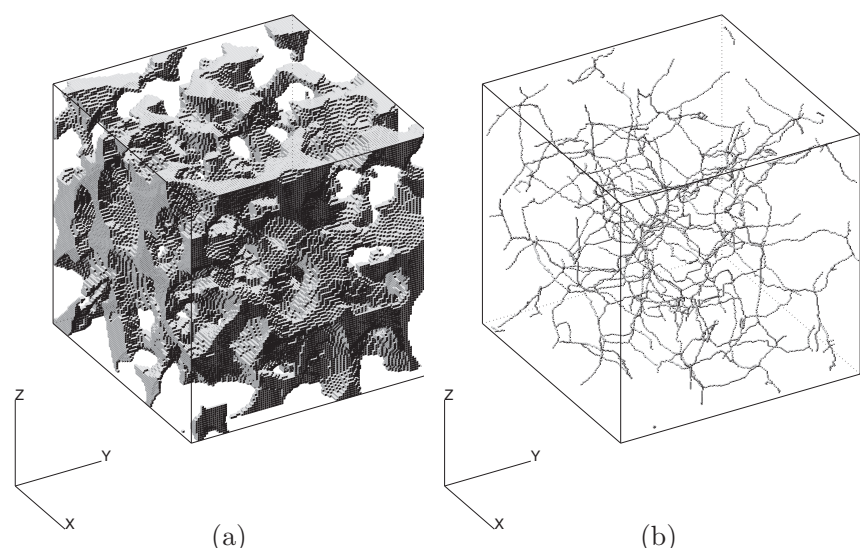

FIG. 8. The pore space in a $125^{3}$ sub-block from the $500^{3} \mathrm{CMT}$ image (a) and its skeleton (b).

$\mathcal{L}_{y}$ [see Eq. (17)]. Second, the conductivity reaches a nearly constant value when the sub-block size exceeds $100 \times 6 \mu \mathrm{m}$, with a standard deviation of the trace of the conductivity tensor smaller than $1 \%$. The conductivity of the whole $500^{3}$ sample was also calculated and found to be equal to $\Sigma / \Sigma_{0}=$ $0.0647,0.0731$, and 0.0672 along the $x, y$, and $z$ directions, respectively.

A second way is to represent the data as functions of porosity [cf. Fig. 10(b)]. Archie laws can be derived for the data depending on the size of the blocks,

$$
\begin{array}{lll}
\left(N_{c}=100\right) & \Sigma / \Sigma_{0}=1.00 \epsilon^{1.81} & (6.8 \%) \\
\left(N_{c}=125\right) & \Sigma / \Sigma_{0}=0.96 \epsilon^{1.79} & (5.3 \%) \\
\left(N_{c}=250\right) & \Sigma / \Sigma_{0}=0.78 \epsilon^{1.66} & (1.7 \%)
\end{array}
$$

These fits were obtained by minimizing the relative deviations between the data and the models. The quadratic mean deviations are given in parentheses. The fits for $N_{c}=100$ and 125 are very close one to another; the fit for $N_{c}=250$ differs slightly, but it is calculated on eight points only and over a limited range of values of porosity. A further analysis of these fits is difficult.

Therefore, these fits should be used with care. They should not be used outside of the porosity range for which they were obtained. No straightforward conclusion can be drawn from the variations of the coefficients.

The conductivity was not measured in Ref. [5], but the authors of Ref. [2] also investigated a Bentheim sandstone with porosity 0.245 and reported a formation factor $F=11.5$, i.e., $\Sigma / \Sigma_{0}=0.087$. This result is shown in Fig. 10(b). It is in the upper range of the numerical results for $(500-\mu)^{3}$ and $(750-\mu)^{3}$ blocks.

\section{B. Permeability of the initial configurations}

Permeability was calculated for the same set of sub-blocks as conductivity. Permeability was systematically calculated along the three directions of space for sub-blocks of various sizes extracted from the $500^{3} \mathrm{CMT}$ image. The results in Fig. 11(a) follow the same trends as those illustrated in Fig. 10(a) for conductivity. Permeability is found to be larger along the $y$ axis. It decreases with the block size and reaches a nearly constant value when the block size exceeds $125 \times 6 \mu \mathrm{m}$. The statistical fluctuations are larger than for conductivity. The standard deviation of the trace of the permeability tensor becomes smaller than $1 \%$ when the block size exceeds $250 \times 6 \mu \mathrm{m}$. Due to the length of the calculations, $K$ was calculated in the whole $500^{3}$ sample only along the $x$ direction. It was found to be equal to $3.38 \mathrm{D}$.

The results are displayed as functions of the porosity of the sub-blocks in Fig. 11(b). Three power laws can be derived for these data depending on the size of the blocks,

$$
\begin{array}{lll}
\left(N_{c}=100\right) & K=275 \epsilon^{2.95} & (22.0 \%), \\
\left(N_{c}=125\right) & K=527 \epsilon^{3.41} & (16.0 \%), \\
\left(N_{c}=250\right) & K=247 \epsilon^{2.86} & (3.7 \%),
\end{array}
$$

where the permeabilities are given in darcy. Figure 11(b) also shows the values measured by Ref. [5], $K_{a, \mathrm{ML}}=2.89 \mathrm{Da}$ and $K_{r}=2.34 \mathrm{Da}$. The axial midlength measurement, which is believed to be the most reliable, is in the lower range of the numerical results for $(500-\mu)^{3}$ and $(750-\mu)^{3}$ blocks.

Finally, power-law relationships can be given between permeability and conductivity,

$$
\begin{array}{lll}
\left(N_{c}=100\right) & K=322\left(\Sigma / \Sigma_{0}\right)^{1.69} & (16.0 \%), \\
\left(N_{c}=125\right) & K=604\left(\Sigma / \Sigma_{0}\right)^{1.93} & (12.0 \%), \\
\left(N_{c}=250\right) & K=365\left(\Sigma / \Sigma_{0}\right)^{1.71} & (2.4 \%) .
\end{array}
$$

Note that (20) results from a direct fit of permeability versus conductivity, but the results are close to that obtained by combining (18) and (19).

The same comments as for conductivity can be made about these two sets of approximate power laws.

\section{Effective elastic coefficients}

Note first that in the mechanical tests of Ref. [5], for both hydrostatic and uniaxial compressions, a nonlinear initial behavior is observed when the load first increases from zero. This can result from the closure of microcracks or from the very compliant Hertzian behavior of quasipunctual grain contacts. Then a linear regime is established for larger loads, beyond about $15 \mathrm{MPa}$. All the measurements reported below are obtained in this linear range. It extends up to $40 \mathrm{MPa}$ for a uniaxial load, with failure at $52 \mathrm{MPa}$. It applies up to the largest load $65 \mathrm{MPa}$ imposed in the hydrostatic test. Hydrostatic tests with a similar Bentheim sandstone by the authors of Ref. [1] showed that the linear behavior extends much farther, with the onset of crushing and pore collapse at $390 \mathrm{MPa}$, corresponding to a volumetric strain about 0.03 .

The present numerical simulations do not take into account the phenomena which cause the initial nonlinear response. They are more representative of the subsequent linear regime in a prestressed state which is probably closer to the actual in situ situation.

A complete series of mechanical tests has been performed in two $100^{3}$ sub-blocks extracted from the $500^{3}$ image and denoted by SB1 and SB2. These tests include the hydrostatic, oedometric, and uniaxial loads described in Sec. II A, with an imposed uniform displacement on the sides of the sample as illustrated in Fig. 2. In addition, hydrostatic tests have 


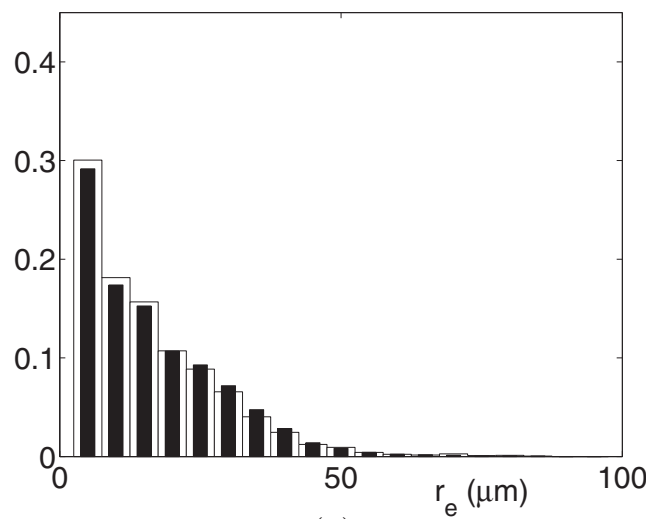

(a)

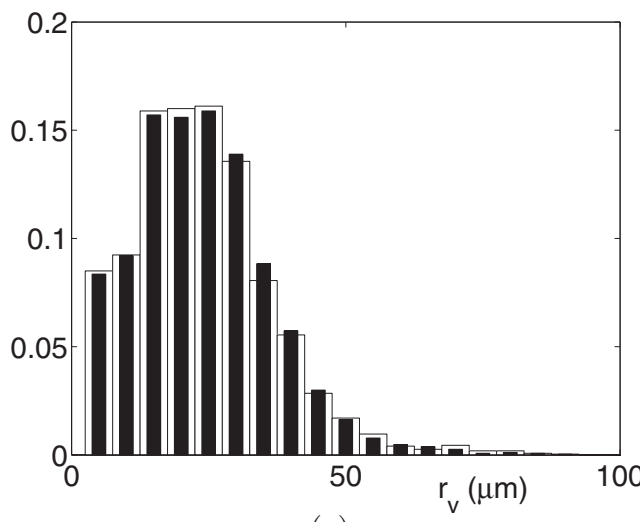

(c)

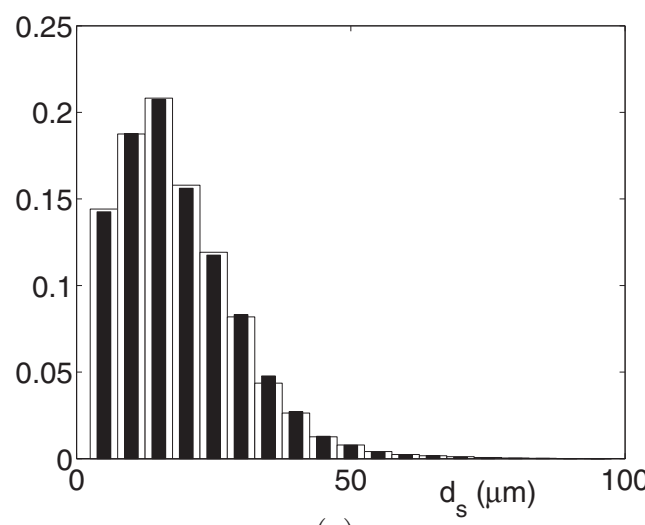

(e)

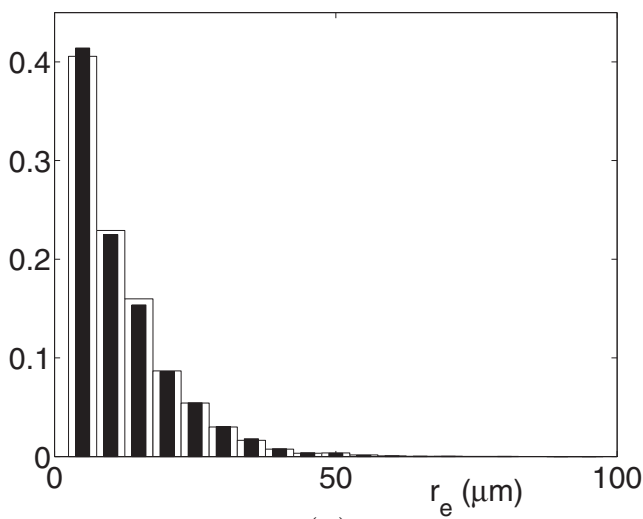

(b)

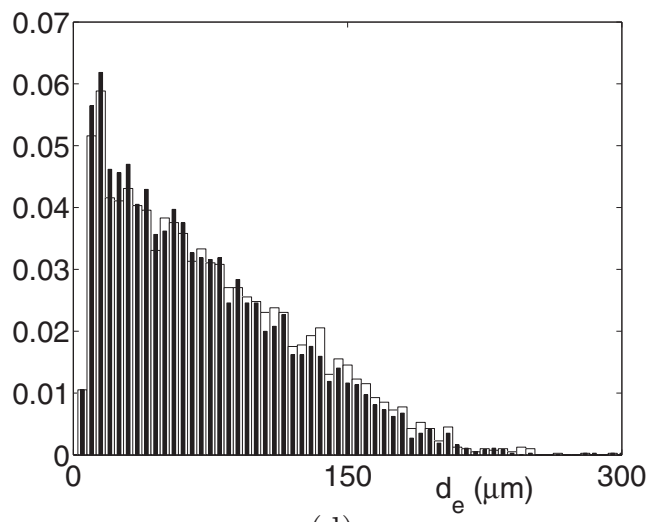

(d)

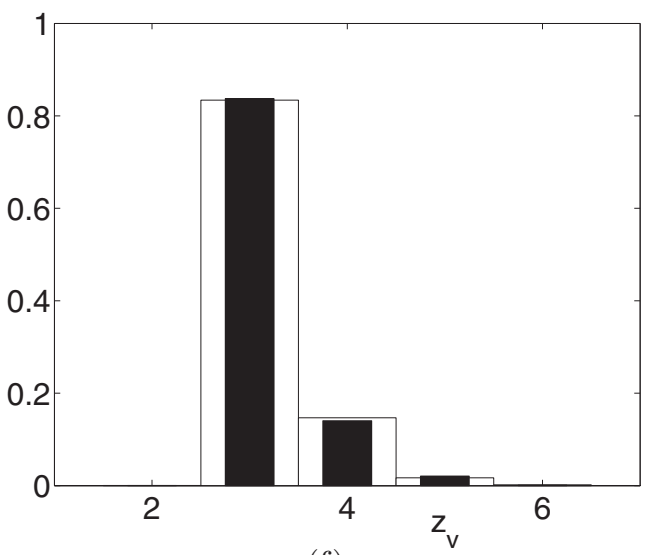

(f)

FIG. 9. Normalized histograms of the critical edge radii $r_{e}$ [number-weighted in (a) or length-weighted in (b)], vertex radii $r_{v}$ (c), edge length $d_{e}$ (d), distance to the solid surface $d_{s}$ (e), and coordination number $z_{v}$ (f). Results for the $250^{3}$ block (white) cut from the 500 $0^{3}$ CMT image and mean over eight nonoverlapping sub-blocks $123^{3}$ (black) cut from the block $250^{3}$.

been simulated in eight $250^{3}$ sub-blocks cut from the $500^{3}$ image. These calculations are much more limited than the ones performed for conductivity and permeability because of their duration as mentioned in Sec. IV A.

We only consider here the initial elastic moduli of the porous medium, i.e., the equations (1) are solved in the linearized, small deformation approximation. Large deformations are addressed in the next Sec. VD.

The effective elastic moduli obtained for SB1 and SB2 are given in Table I. All the moduli are found to be slightly smaller in SB2, which is expected since its porosity is slightly larger than that of SB1. However, the uniaxial test yields identical Poisson ratios for the two samples.

Note that due to their small size, both samples present some anisotropy. In particular for SB2, the diagonal components of the stress tensor induced by an isotropic deformation differ by $\pm 4 \%$, and the mean stresses $\left\langle\sigma_{x x}\right\rangle$ and $\left\langle\sigma_{y y}\right\rangle$ in the oedometric test with compression along $z$ differ by $8 \%$. Aside from these two cases, all the symmetries expected in each load case if isotropy is assumed are satisfied within a $\pm 1.5 \%$ interval.

Table I gives the elastic moduli obtained by (2) from the sample responses in the three standard mechanical tests and, 


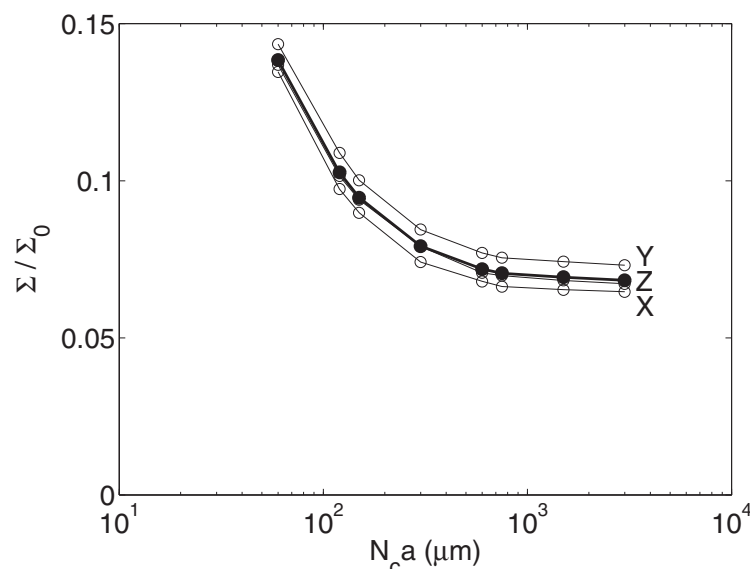

(a)

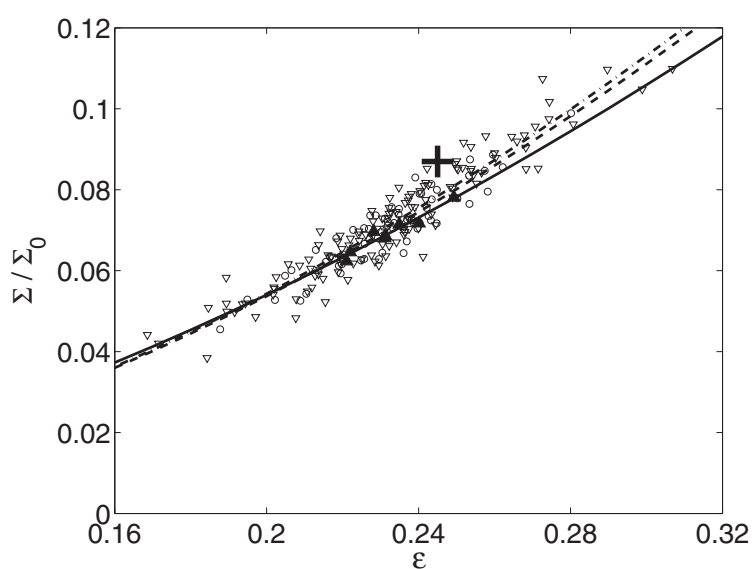

(b)

FIG. 10. (a) The conductivity vs. the size of the sub-blocks cut from the $500^{3}$ CMT image, along the $x, y$, and $z$ directions (o) and in average over the three axes $(\bullet)$. (b) The conductivity averaged over the three directions as function of porosity. Data are for $N_{c}=100(\nabla), 125$ (०), and 250 ( $\Delta$ ). The dash-dotted, broken, and solid lines, respectively, are the corresponding power laws (18). The black cross corresponds to the measurement of Ref. [2].

for illustration, the values of the $K_{e}, E_{e}$, and $v_{e}$ deduced from the Lamé coefficients if isotropy is assumed. Small differences are observed which never exceed $5 \%$.

Of course, statistical uncertainty is also associated with this limited size, and the results for SB1 and SB2 should only be regarded as general trends. Simulations on larger samples have been conducted for the hydrostatic test, by splitting the $500^{3}$ sample into eight $250^{3}$ sub-blocks.

The resulting effective bulk moduli are plotted in Fig. 12 as functions of the porosity. The average value of $K_{e}$ is 0.499 $K_{s}$ with fluctuations linearly related to the deviations of the sub-block porosities from the overall block porosity,

$$
\frac{K_{e}}{K_{s}} \approx 0.50-1.7(\epsilon-0.232) \text {. }
$$

This linear relationship can be used to predict the fluctuations of $K_{e}$ on a millimetric scale, induced by the porosity fluctuations which have been quantified in Sec. IV B (see Fig. 5).
It is interesting to compare this result with classical variational bounds. The best possible bounds for the bulk modulus of composite media have been established by Hashin and Shtrikman [25] when isotropy is assumed and the only available geometrical knowledge is the porosity. Higher-order and tighter bounds can be stated when additional morphological information is used, such as those formulated by Milton [26]. When the material is composed of a solid and a void phase, the lower bound vanishes and the upper bound is given by

$$
\frac{K_{e}}{K_{s}} \leqslant \frac{1-\epsilon}{1+\frac{1+v_{s}}{2\left(1-2 v_{s}\right)} \frac{\epsilon}{1-\zeta}},
$$

where $\zeta$ is a structural parameter. Hashin and Shtrikman's upper bound is obtained by setting $\zeta=0$.

Visual examination of Fig. 1(a) suggests that representing the solid phase by randomly located fully penetrable spheres

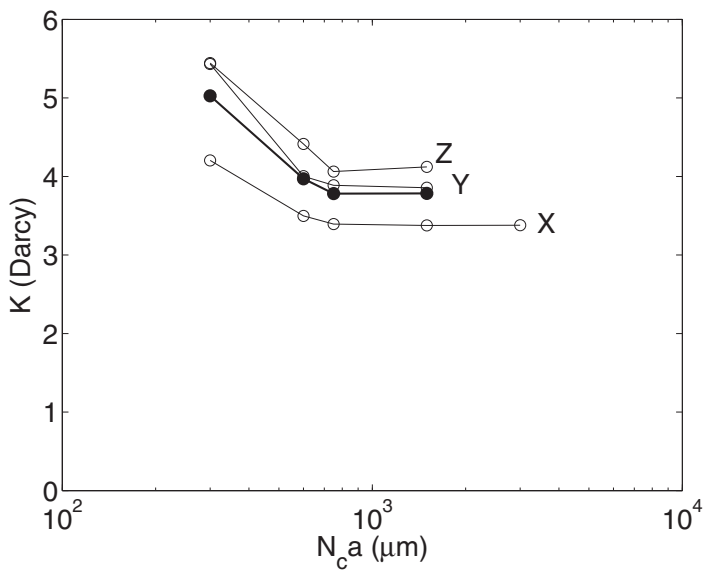

(a)

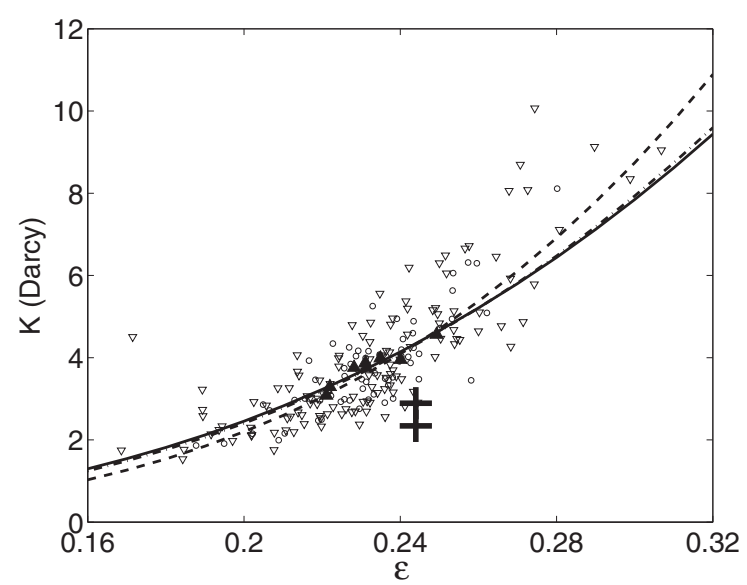

(b)

FIG. 11. (a) The permeability vs. the size of the sub-blocks cut from the 500 3 CMT image, along the $x, y$, and $z$ directions ( $\circ$ ) and the average over the three directions $(\bullet)$. (b) The permeability averaged over the three directions as a function of porosity. Data are for $N_{c}=100$ $(\nabla), 125(\circ)$, and $250(\mathbf{\Delta})$. The dash-dotted, broken, and solid lines, respectively, are the corresponding power laws (19). The black crosses correspond to the measurements of $K_{a, \mathrm{ML}}$ and $K_{r}$ by Ref. [5]. 
TABLE I. The main characteristics of sub-blocks SB1 and SB2. Their size, porosity, and effective transport coefficients; their effective elastic moduli obtained as stated in Eq. (2), and for $K_{e}, E_{e}$, and $v_{e}$, as deduced from the Lamé coefficients $\lambda_{e}$ and $\mu_{e}$ obtained by (2b).

\begin{tabular}{lcc}
\hline \hline & SB1 & SB2 \\
\hline Size & $100 \times 6 \mu \mathrm{m}$ & $100 \times 6 \mu \mathrm{m}$ \\
$\epsilon$ & 0.226 & 0.233 \\
$\Sigma / \Sigma_{0}$ & 0.069 & 0.074 \\
$K(\mathrm{Da})$ & 2.75 & 3.29 \\
$K_{e} / K_{s}$ from $(2 \mathrm{a})$ & 0.517 & 0.500 \\
$K_{e} / K_{s}$ from $\left(\lambda_{e}, \mu_{e}\right)$ & 0.508 & 0.476 \\
$E_{e} / E_{s}$ from $(2 \mathrm{c})$ & 0.461 & 0.430 \\
$E_{e} / E_{s}$ from $\left(\lambda_{e}, \mu_{e}\right)$ & 0.468 & 0.442 \\
$\nu_{e}$ from $(2 \mathrm{c})$ & 0.099 & 0.099 \\
$\nu_{e}$ from $\left(\lambda_{e}, \mu_{e}\right)$ & 0.104 & 0.101 \\
$\lambda_{e} / \lambda_{s}$ from $(2 \mathrm{~b})$ & 0.729 & 0.669 \\
$\mu_{e} / \mu_{s}$ from $(2 \mathrm{~b})$ & 0.454 & 0.429 \\
\hline \hline
\end{tabular}

is a reasonable tentative model. It indeed proved successful in reproducing the transport and mechanical properties of the real material when applied with a polydisperse grain size distribution [6]. However, we use here the parameter $\zeta \approx 0.54$ for monodisperse spheres obtained by interpolation from the tabulated values of Ref. [27]. After linearization in the present range of porosity, Hashin and Shtrikman's and Milton's upper bounds read

$$
\begin{aligned}
& \frac{K_{e}}{K_{s}} \leqslant 0.67-1.24(\epsilon-0.232) \quad(\zeta=0) \\
& \frac{K_{e}}{K_{s}} \leqslant 0.585-1.36(\epsilon-0.232) \quad(\zeta=0.54)
\end{aligned}
$$

The numerical result (21) conforms with these theoretical predictions. $K_{e}$ is smaller than these bounds, and its dependence on porosity is steeper but as is often the case the upper bound is a reasonable first guess for the elastic coefficient, especially when the variational argument is combined with the penetrable sphere geometrical model.

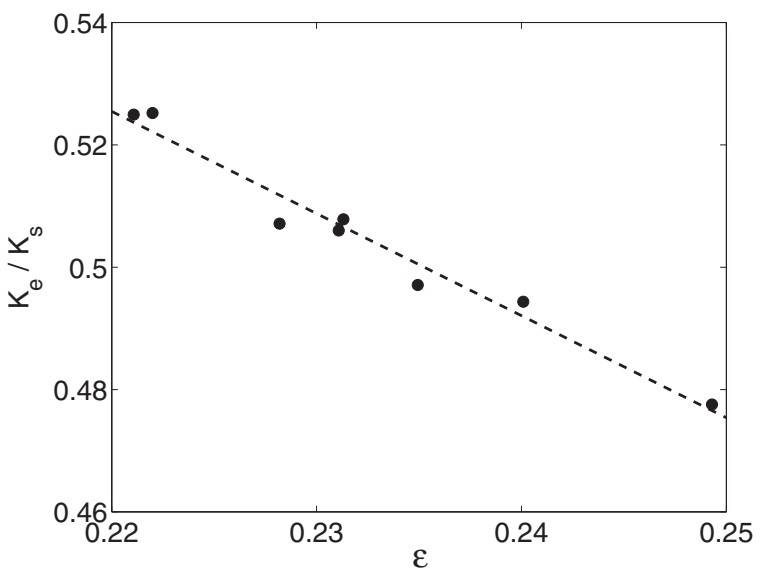

FIG. 12. The initial bulk modulus in hydrostatic compression for the 8 sub-blocks $250^{3}$ of the $500^{3}$ CMT image, as a function of porosity. The broken line corresponds to the linear fit (21).
Let us finally compare these numerical results with the experimental measurements. This requires the knowledge of the solid bulk modulus $K_{s}$, which is not reported in Ref. [5] but can be found in Ref. [28]. It was deduced from the combination of tests where the pore and confining pressures were varied independently, which yielded

$$
K_{s}=16.2 \mathrm{GPa}, \quad K_{e}=6.96 \mathrm{GPa}, \quad K_{e} / K_{s}=0.430 .
$$

The ratio $K_{e} / K_{s}$ is $10 \%$ smaller than predicted by the application of (21) with the porosity 0.244 of the sample in Ref. [28], which yields 0.479 .

Consider now the data deduced from the uniaxial tests. The value of $K_{s}$ in (24) together with the Poisson ratio $v_{s}=0.07$ used in the numerical calculations correspond to a Young modulus $E_{s}=41.8 \mathrm{GPa}$. Hence, the results in Table I yield $E_{e}=18-19 \mathrm{GPa}$, which is significantly larger than the measured value $10.3 \mathrm{GPa}$ [5]. Conversely, the Poisson ratio $v_{e} \approx 0.1$ is much smaller than the measured value $\approx 0.2$.

These discrepancies can originate in the value of the solid Poisson ratio adopted in the numerical model. We have no direct indication of this parameter in the real material. The value $v_{s}=0.07$ corresponds to $\alpha$ quartz [29]. It is anomalously low compared to other minerals whose ratios are generally of the order of 0.2 , but this peculiarity is well known (see, e.g., Refs. [30,31]). The response in a hydrostatic test is only weakly sensitive to $v_{s}$, in terms of $K_{e} / K_{s}$, and even totally unsensitive in the case of an unconsolidated granular material, which explains the relatively good agreement of (21) and (24).

Conversely, the response to uniaxial load is obviously much more sensitive to the solid Poisson ratio. If $v_{s}$ is tentatively set to 0.2 while keeping $K_{s}=16.2 \mathrm{GPa}, E_{s}=29.2 \mathrm{GPa}$ and $E_{e}$ becomes $13 \mathrm{GPa}$, in much better agreement with the measurement. But, of course, this is only a gross corrective action, since an exact prediction requires us to solve the local equations (1) again with the modified Poisson ratio.

\section{Properties of the deformed samples}

We consider here the changes in the material properties induced by a mechanical load for larger deformations. The first and most obvious ones are the volume changes of the solid and void spaces and the porosity variations. We also consider the modifications of the permeability. Both effects are of primary importance for hydromechanical coupling. In addition, we quantify the variations of some of the elastic properties of the porous medium beyond the small deformation regime.

Performing conductivity calculations in the deformed state was not found to be useful since there were no experimental data to compare with.

In order to achieve large deformations, successive incremental steps of small deformations are applied. Hence, the elastostatic equations (1) can still be linearized, provided that they are discretized on a deformed mesh, which takes into account the displacements caused by previous steps. These calculations have been performed for the three kinds of loads, in the sub-blocks SB1 and SB2, with five successive increments of $0.5 \%$ deformation. 


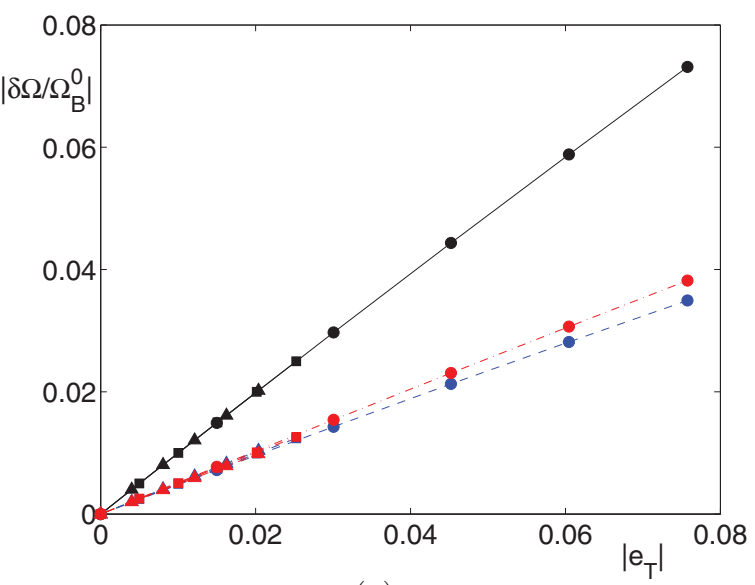

(a)

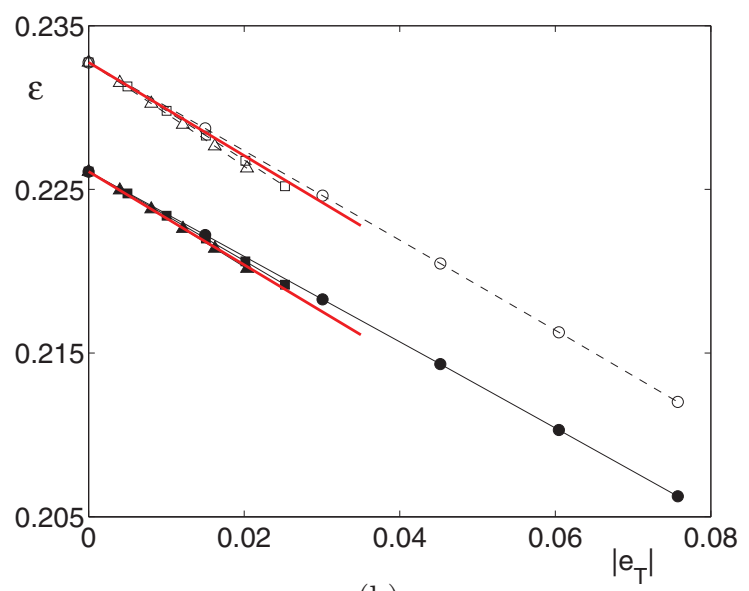

(b)

FIG. 13. (Color online) The volume changes $\delta \Omega_{B}$ (black, solid line), $\delta \Omega_{s}$ (red, dash-dotted line), and $\delta \Omega_{p}$ (blue, dashed line) normalized by $\Omega_{B}^{0}$ in SB1 (a) and the variations of the porosity in SB1 (solid lines and symbols) and SB2 (broken lines and open symbols) (b) as functions of $e_{T}$. Data are for isotropic deformation (o), oedometric test ( $\square$ ), and unaxial test ( $\Delta$ ). The thick red lines in (b) show the slope of the fit (37) of the experimental data in Ref. [5].

\section{Volumes and porosity}

Let us introduce first some notations. Recall that the bulk volume $\Omega_{B}$ of the porous medium is decomposed into the solid volume $\Omega_{s}$ and the pore volume $\Omega_{p}$. Their initial values are denoted by a superscript 0 , and their variations induced by a deformation are denoted by $\delta \Omega$. For instance, $\Omega_{p}=\Omega_{p}^{0}+$ $\delta \Omega_{p}$. Moreover, define $e_{T}$ as the trace of the strain tensor

$$
e_{T}=\left\langle e_{x x}\right\rangle+\left\langle e_{y y}\right\rangle+\left\langle e_{z z}\right\rangle .
$$

For later use, define in a similar way $\sigma_{T}$ as the trace of the stress tensor. If the deformations are small, $e_{T}$ represents the relative variation of the bulk volume,

$$
\begin{aligned}
\frac{\delta \Omega_{B}}{\Omega_{B}} & =\left(1+\left\langle e_{x x}\right\rangle\right)\left(1+\left\langle e_{y y}\right\rangle\right)\left(1+\left\langle e_{z z}\right\rangle\right)-1 \\
& \approx\left\langle e_{x x}\right\rangle+\left\langle e_{y y}\right\rangle+\left\langle e_{z z}\right\rangle=e_{T} .
\end{aligned}
$$

Note that the simulations have been conducted with compressive deformations. Hence, $e_{T}, \sigma_{T}$, and the volume variations are all negative. For readability, their absolute values are plotted in the following figures.

The volume variations $\delta \Omega_{B}, \delta \Omega_{s}$, and $\delta \Omega_{p}$ normalized by $\Omega_{B}^{0}$ are plotted in Fig. 13(a) as functions of $e_{T}$ for the three kinds of tests in SB1. A similar picture is obtained when considering SB2. The results for all the tests are approximately gathered along single curves. A slight negative inflection of these curves is visible beyond $e_{T} \approx 0.03$, when the approximation (26) becomes inaccurate. The solid and pore volumes vary by very similar amounts. Since $\Omega_{s}^{0}$ and $\Omega_{p}^{0}$ different so much, this means that their relative variations differ. It is therefore natural to examine the porosity variations.

The evolution of $\epsilon$ with $e_{T}$ is shown in Fig. 13(b) for the three tests in SB1 and SB2. The porosity decreases linearly with $e_{T}$, according to

$$
\begin{array}{lll}
\delta \epsilon=0.262 e_{T} & (\mathrm{SB} 1), & \delta \epsilon=0.274 e_{T} \\
\delta \epsilon=0.274 e_{T} & (\mathrm{SB} 1), & \delta \epsilon=0.300 e_{T} \\
\delta \epsilon=0.291 e_{T} & (\mathrm{SB} 1), & \delta \epsilon=0.318 e_{T}
\end{array}
$$

for the isotropic, oedometric, and uniaxial deformations, respectively. The correlation coefficient is larger than 0.999 95 in all cases.

Recall that for small isotropic deformations, the porosity increment induced by a macroscopic strain with trace $e_{T}$ is

$$
\delta \epsilon=\left(1-\epsilon-\frac{K_{e}}{K_{S}}\right) e_{T} .
$$

This is the application of Eq. (28) of Ref. [32] to a dry porous medium with uniform solid properties, expressed in terms of the macroscopic strain instead of the confining pressure. This result is valid for any linearly elastic porous medium. This yieds 0.257 and 0.267 with the values of $\epsilon$ and $K_{e}$ in Table I for SB1 and SB2, respectively, in very good agreement with (27a). The slight differences result from the finite size of the samples, which make their elastic coefficients apparent rather than effective. When applied with the overall porosity $\epsilon=0.232$ and the bulk modudus $K_{e} / K_{s}=0.50$ [see Eq. (21)], (28) yields

$$
\delta \epsilon=0.268 e_{T}
$$

This is also a good prediction of the numerical results (27a).

Note that (28) is also valid for any anisotropic deformation, provided that the porous medium elastic properties are macroscopically isotropic and the results [(27b) and (27c)] are indeed close to (29). However, the slightly larger porosity variations in the oedometric and uniaxial tests can be explained by use of a stronger form of (28) presented below.

The porosity variation is given by

$\delta \epsilon=\frac{\Omega_{p}}{\Omega_{B}}-\frac{\Omega_{p}^{0}}{\Omega_{B}^{0}}=\frac{\delta \Omega_{p}}{\Omega_{B}}-\epsilon^{0} \frac{\delta \Omega_{B}}{\Omega_{B}} \approx \frac{\delta \Omega_{p}}{\Omega_{B}^{0}}-\epsilon^{0} \frac{\delta \Omega_{B}}{\Omega_{B}^{0}}$,

where the last form is the first-order approximation for small deformations. The last term in (30) is exactly $\epsilon^{0} e_{T}$. If the solid material has uniform properties, it can be shown by application of the Maxwell-Betti reciprocal theorem and by superposition 
arguments that

$$
\frac{\delta \Omega_{p}}{\Omega_{B}^{0}}=\left[\mathbf{I}-\frac{1}{3 K_{s}}\left\langle\boldsymbol{\sigma}_{1}\right\rangle\right]:\langle\mathbf{e}\rangle
$$

where $\left\langle\sigma_{1}\right\rangle$ is the mean stress tensor resulting from a unit spherical strain of the porous medium. Hence, for small deformations

$$
\delta \epsilon=\left[\left(1-\epsilon^{0}\right) \mathbf{I}-\frac{1}{3 K_{s}}\left\langle\boldsymbol{\sigma}_{1}\right\rangle\right]:\langle\mathbf{e}\rangle .
$$

Equations (31) and (32) are strong and general results, whereby the porosity change under an arbitrary strain can be deduced from the stress response under a hydrostatic load, without any hypothesis aside from the uniformity of the solid properties. If the medium is macroscopically isotropic, $\left\langle\boldsymbol{\sigma}_{1}\right\rangle=3 K_{e} \mathbf{I}$ and (32) reduces to (28).

The values of $\left\langle\sigma_{1}\right\rangle$ obtained numerically in SB1 and SB2 are

$$
\begin{aligned}
& \left\langle\sigma_{1}\right\rangle=\left(\begin{array}{rrr}
1.571 & -0.021 & -0.001 \\
-0.021 & 1.561 & -0.031 \\
-0.001 & -0.031 & 1.523
\end{array}\right) K_{s} \quad(\mathrm{SB} 1), \\
& \left\langle\sigma_{1}\right\rangle=\left(\begin{array}{rrr}
1.531 & 0.014 & -0.000 \\
0.014 & 1.539 & 0.021 \\
-0.000 & 0.021 & 1.428
\end{array}\right) K_{s} \quad(\mathrm{SB} 2) .
\end{aligned}
$$

Direct application of (32) with (33) to the oedometric test where $\left\langle e_{x x}\right\rangle$ and $\left\langle e_{y y}\right\rangle$ are imposed to be zero yields

$$
\delta \epsilon=0.266 e_{T} \quad(\mathrm{SB} 1), \quad \delta \epsilon=0.291 e_{T} \quad(\mathrm{SB} 2) .
$$

This is in good agreement with (27b). In particular, in the most anisotropic sample SB2, both (27b) and (34) predict a response $9 \%$ larger in the oedometric test than in the isotropic load. The success of this comparison is an important conclusion since in situ deformations induced by surface loads or subsidence are likely to occur with $\left\langle e_{x x}\right\rangle \approx\left\langle e_{y y}\right\rangle \approx 0$. Note that only the knowledge of the $z z$ component of $\left\langle\sigma_{1}\right\rangle$ is required in this situation.

Application of (32) is less straightforward for the uniaxial test since $\left\langle e_{x x}\right\rangle$ and $\left\langle e_{y y}\right\rangle$ are not known a priori. However, the knowledge of the effective Poisson ratio $v_{e}$ can be used to circumvent this, since the mean strain tensor is given by

$$
\langle\boldsymbol{e}\rangle=\left(\begin{array}{ccc}
-v_{e} /\left(1-2 v_{e}\right) & 0 & 0 \\
0 & -v_{e} /\left(1-2 v_{e}\right) & 0 \\
0 & 0 & 1 /\left(1-2 v_{e}\right)
\end{array}\right) e_{T}
$$

This yields eventually for the uniaxial test, with $v_{e}=0.10$ (see Table I),

$$
\delta \epsilon=0.270 e_{T} \quad(\mathrm{SB} 1), \quad \delta \epsilon=0.300 e_{T} \quad(\mathrm{SB} 2) .
$$

The agreement with (27c) is slightly less accurate than for the oedometric test, since (36) predicts a response in SB2 larger by $12 \%$ than for an isotropic load, instead of $16 \%$ in (27c), but the trend is clearly captured.

Let us now compare these results with the experimental measurements of the porosity variations under load. Data are available in Ref. [5] only for the hydrostatic test. It should be noted that the porosity changes are quantified in Ref. [5] by dividing the pore volume change by the initial sample volume, whereas we evaluate it here from the actual pore volume fraction in the current sample volume [see Eq. (30)]. Therefore, the data of Ref. [5] have been reprocessed to allow a direct comparison. A linear fit of the data in the range 15-65 $\operatorname{MPa}\left(\right.$ i.e., $\left.\left|e_{T}\right|>0.0044\right)$ yields

$$
\epsilon=0.241-0.285\left(\left|e_{T}\right|-0.0044\right) \text {. }
$$

The comparison is very satisfactory since the coefficients in the numerical results (27a) and in the prediction (29) differ only by a few percentages from that in (37). The slope of the dependence in (37) is indicated in Fig. 13(b) in comparison with the numerical results.

\section{Permeability}

The permeability has been calculated in SB1 and SB2 for $0.5 \%, 1 \%$, and $2 \%$ deformation rates in hydrostatic, oedometric, and uniaxial tests. The $K_{x x}$ component was calculated in all cases. $K_{z z}$ was also calculated for the uniaxial load.

It was checked that in the investigated range the calculated permeability is not sensitive to the nonlinearity introduced by the nonvanishingly small deformations. For instance, the permeabilities in SB1 isotropically deformed by $2 \%\left(e_{T} \approx\right.$ 0.06 ) either in a single step or by successive increments differ by less than $0.1 \%$.

The results are presented in Fig. 14 as functions of the porosity and of the strain $e_{T}$. Note that $K_{z z}$ of the initial configurations is larger than $K_{x x}$. Hence, the two sub-blocks present the same permeability anisotropy as the whole sample [see Fig. 11(a)]. Nearly linear decreases are observed in all cases. The evolution of $K_{x x}$ is almost identical in the hydrostatic and oedometric compressions [Fig. 14(a)]. However, $K_{x x}$ and especially $K_{z z}$ decrease faster with $e_{T}$ in the uniaxial test. This difference is slightly lessened in the plot as a function of $\epsilon$ in Fig. 14(b), because $\epsilon$ also varies faster in the uniaxial test than in the two other ones [see Eq. (27)], but it is still significant.

A rough modelization of the permeability variations is possible by using the relationship (19) between $K$ and $\epsilon$ and by combining it with the prediction (29) of the porosity increment induced by the strain. We choose here (19b), which is based on a data set larger than that for the $250^{3}$ sub-blocks and less scattered than that for the $100^{3}$ sub-blocks [see Fig. 11(b)]. This yields

$$
\frac{\delta K}{K}=3.31 \frac{\delta \epsilon}{\epsilon}=\frac{0.890}{\epsilon} e_{T} .
$$

These predictions are plotted in Fig. 14 and found to be in surprisingly good agreement with the numerical data for the hydrostatic and oedometric loads. The model is less successful in representing the steeper variations of the permeability in the uniaxial test, especially for $K_{z z}$.

It is difficult to assess whether this is due solely to the anisotropy of the macroscopic deformation in this particular test or also to its interplay with the anisotropy of the medium itself, which has been detected in the foregoing in its geometrical and transport properties. More extensive calculations as well as a local morphological analysis of the 

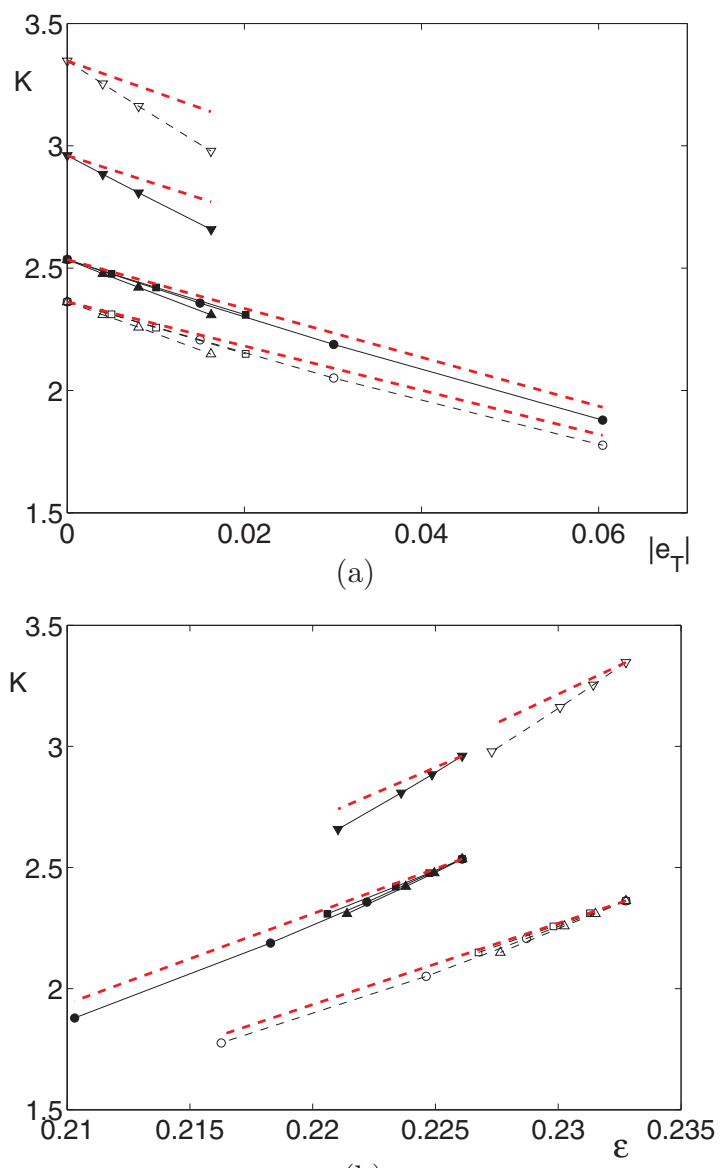

(b)

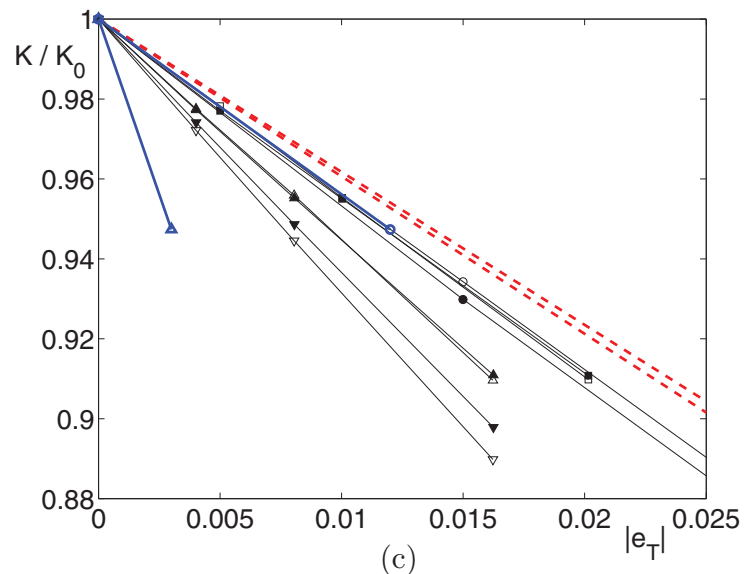

FIG. 14. (Color online) The permeability $K$ in darcy in SB1 (solid lines and symbols) and SB2 (broken lines and open symbols) as a function of the strain $e_{T}$ (a) and of the porosity $\epsilon$ (b), and the permeability normalized by its value at rest $K / K_{0}$ as a function of the strain $e_{T}$ (c). Numericad data are for isotropic deformation (o), oedometric test $(\square)$, and unaxial test along the $x(\Delta)$ and $z(\nabla)$ directions. The red broken lines are the predictions (38). The solid blue lines in (c) correspond to (40).

pore space deformations are required to tackle this interesting question.

Note finally that the success of the fits (19) in accounting for the permeability variations under strain is by no means obvious and possibly fortuitous. They represent spatial variations which originate in some kind of geometrical fluctuations, probably in most part in varying degrees of consolidation with possible impact on the connectivity of the pore space. On the other hand, porosity varies under strain in a different way; for instance, a moderate compression does not affect the pore space connectivity.

For a comparison with the experimental measurements, we use the most reliable and best documented data available in Refs. [5,28]. A definite linear decay of the axial permeability $K_{a, \mathrm{ML}}$ was observed during the hydrostatic compression, but the evolution of the radial permeability $K_{r}$ was unclear, because of fluctuations. During the uniaxial test, only the full length axial permeability $K_{a, \mathrm{FL}}$ was measured, but it is less reliable than $K_{a \text {,ML }}$ because of end effects. However, a linear decay of $K_{r}$ was clearly identified. The two best data sets can be represented by the fitted relationships

$$
\begin{aligned}
K_{a, \mathrm{ML}} & =2.89[1-0.82 P] \quad \text { (hydrostatic), } \\
K_{r} & =2.15[1-1.2 P] \quad \text { (uniaxial), }
\end{aligned}
$$

where permeability is expressed in darcy and the applied load $P$ in GPa. Note that the value $2.15 \mathrm{Da}$ of $K_{r}$ from the value 2.34 mentioned earlier because the uniaxial test was conducted after the sample had undergone other load cycles.

Again, the comparison with the numerical calculations is made in terms of the permeability variations as functions of the volumetric strain instead of the applied load. Therefore, (39) is reformulated as

$$
\begin{aligned}
K_{a, \mathrm{ML}} & =2.89\left[1-4.4\left|e_{T}\right|\right] \quad \text { (hydrostatic), } \\
K_{r} & =2.15\left[1-19\left|e_{T}\right|\right] \quad \text { (uniaxial). }
\end{aligned}
$$

These fits are compared to the numerical data in Fig. 14(c). Note that the lines representing (40) are drawn over the actual range of imposed deformation in the experiments. The agreement is excellent for the hydrostatic load. Conversely, it is very poor for the uniaxial load. The calculations underpredict by a factor of about 3 the permeability decrease. Again, this can be a consequence of an erroneous Poisson ratio for the solid material, which directly affects the deformation and therefore the permeability response under uniaxial load. This might also be due to a possible degradation of the sample since the uniaxial test was conducted after the others.

\section{Elastic coefficients}

The variations of the elastic coefficients beyond the small deformation range have not been investigated in depth, because even with the very large deformation rates applied here which cannot be performed experimentally without breaking the sample, these variations are found to be very small. Hence, the geometrical effect is probably negligible in practice, compared to other more important sources of nonlinearity which occur much sooner, such as nonlinear point contact behaviors, damage, or the onset of plasticity, which are not accounted for in the present model.

We provide a single example regarding the isotropic strain and the bulk modulus. The trace $\sigma_{T}$ of the stress tensor is plotted in Fig. 15(a) as a function of $e_{T}$ for SB1 and SB2. There is a slight negative inflection to the curves, but it is barely visible. It is more easily quantified in the plot in Fig. 15(b) 


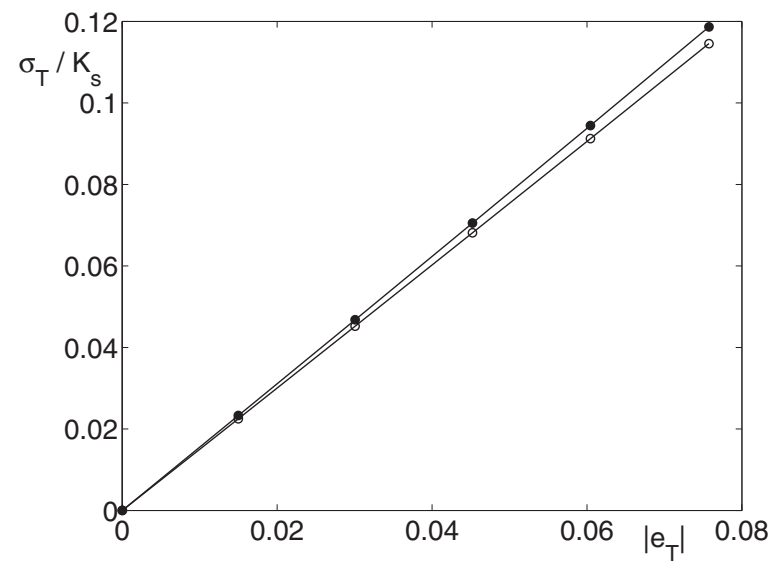

(a)

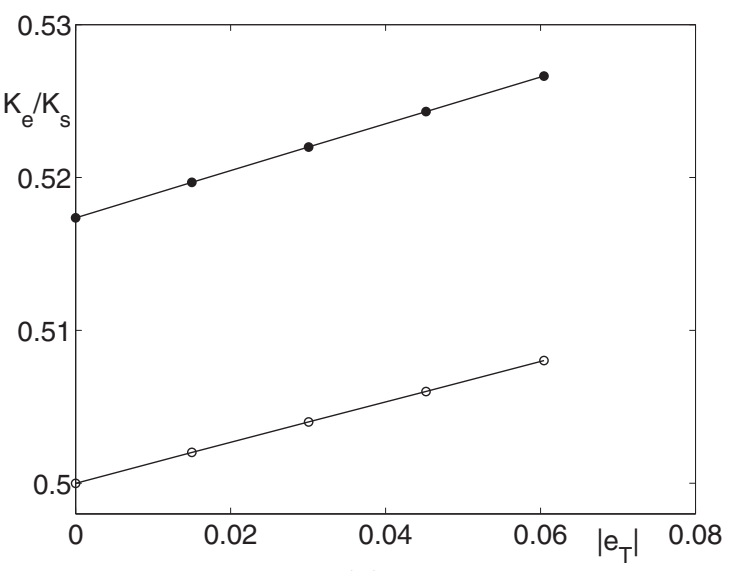

(b)

FIG. 15. The trace $\sigma_{T} / K_{s}$ of the stress tensor (a) and the bulk modulus $K_{e} / K_{s}$ (b) in successive deformed states as functions of $e_{T}$ for an isotropic compression in SB1 (solid lines and symbols) and SB2 (broken lines and open symbols).

of the ratio $\delta \sigma_{T} / 3 \delta e_{T} K_{s}$ which corresponds to the value of $K_{e} / K_{s}$ in the successive deformed states. It only increases by $1.8 \%$ and $1.6 \%$ in SB1 and SB2, respectively, between the initial configuration and the last one with $e_{T} \approx-0.06$.

Note that the naive argument which combines the dependence (21) of $K_{e}$ on the porosity and the variation (29) of $\epsilon$ with the strain $e_{T}$ overestimates the variations of $K_{e}$ by a factor of about 3 . This is because (21) corresponds to the strong dependence of $K_{e}$ on the consolidation, which strengthens the grain contacts, whereas the strain which induces the porosity variations described by (29) has no such effect.

No quantitative comparison can be made here with experimental data. Although a slight increasing trend of the bulk modulus under load seems to exist, it is difficult to quantify.

\section{CONCLUDING REMARKS}

The properties of the initial configurations and under load of a Bentheim sandstone sample have been investigated by direct numerical simulations based on a microtomographic image of its microstructure. The use of unstructured tetrahedral meshes which can follow continuously the deformation of the medium is a significant improvement on the fixed cubic voxels which were used in Ref. [9] for the mechanical deformation of fractures. When addressing large deformations, the elasticity and the Stokes equations are solved on the progressively deformed tetrahedral meshes corresponding to successive incremental loads. This results in a significant improvement of the precision.

The comparison of the numerical results with the available experimental data is very good for isotropic loads. They reproduce well the behavior observed experimentally in the linear elastic range beyond the initial very compliant deformation when the load first increases from zero, i.e., in the prestressed state which probably corresponds to the in situ conditions. The results are also in good agreement with the applicable theoretical predictions.

This comparison is less satisfactory for the oedometric and uniaxial loads. This probably results in great part from the uncertainty about the Poisson ratio of the solid material, which has a greater influence on the mechanical response in these situations than in hydrostatic deformations.

The direct resolution of the governing microscale equations in the real sample geometry imaged by CMT can be tentatively regarded as a reference for the assessment of various conceptual models intended to mimic the properties of the real material. Table II compares various quantities as obtained from experiments, from the present direct simulations, and from several models. In three of them [6], numerical samples are reconstructed either by random insertion of penetrable spheres with a polydisperse size distribution conditioned in two different ways by measured geometrical characteristics $\left(\mathrm{PSM}_{g}\right.$ and $\mathrm{PSM}_{R}$ ) or by thresholding a random Gaussian field generated according to the measured two-point correlation function (TGF). In the last one (PNM), a pore network model is built, deduced from the skeleton of the pore space extracted from the CMT image, and composed of pores separated by throats [5].

Comparison of the numerical and experimental results should be taken with caution, since the experiments have been conducted with different or larger rock samples. However, the same CMT image has been used for the present direct simulations and for the conditioning of all the numerical models.

TABLE II. The conductivity, permeability, effective bulk modulus, porosity and permeability decrease coefficients under isotropic load, as determined from experiments [2,5], by direct simulations in the CMT image, by direct simulations in samples reconstructed by use of two penetrable sphere models and thresholded Gaussian fields [6] and by calculations in pore network models [5].

\begin{tabular}{lccccc}
\hline \hline & $\Sigma / \Sigma_{0}$ & $K(\mathrm{Da})$ & $K_{e} / K_{s}$ & $\delta \epsilon / e_{T}$ & $\delta K / K e_{T}$ \\
\hline Experiments [2,5] & 0.087 & $2.9 / 2.3$ & 0.430 & 0.285 & $4.4 / 19$ \\
CMT & 0.069 & 3.7 & 0.499 & 0.268 & 3.8 \\
PSM $_{g}$ [6] & 0.078 & 4.2 & 0.433 & 0.349 & - \\
PSM $_{R}$ [6] & 0.074 & 4.3 & 0.456 & 0.321 & - \\
TGF [6] & 0.036 & 2.2 & 0.580 & 0.197 & - \\
PNM [5] & - & 0.87 & - & 0.156 & 3.9 \\
\hline \hline
\end{tabular}


The two PSM models are nearly equivalent and slightly overestimate the conductivity and permeability of the initial configurations, while they underestimate the bulk modulus and therefore exaggerate the porosity variation induced by an isotropic load. TGF model has just the opposite behavior. These two classes of models bracket the truth, possibly because TGF ignores the underlying granular nature of the sandstone, while PSM does not explicitly account for consolidation. The pore network model PNM is only intended to represent the flow properties. Thus, only its prediction for permeability can be compared, and it is significantly smaller than all other predictions or measurements. However, the relative permeability decrease due to isotropic compression is in good agreement with the results of the present direct calculations and with the measurements in the axial direction.

\section{ACKNOWLEDGMENT}

This work was partly supported by a grant of the IFP Energies Nouvelles whose support is gratefully acknowledged.
[1] E. Klein, P. Baud, T. Reuschlé, and T.-f. Wong, Phys. Chem. Earth (A) 26, 21 (2001).

[2] L. Louis, C. David, and P. Robion, Tectonophysics 370, 193 (2003).

[3] L. Louis, C. David, V. Metz, P. Robion, B. Menéndez, and C. Kissel, Int. J. Rock Mech. Min. Sci. 42, 911 (2005).

[4] P. M. Benson, P. G. Meredith, E. S. Platzman, and R. E. White, Int. J. Rock Mech. Min. Sci. 42, 890 (2005).

[5] J. Dautriat, N. Gland, S. Youssef, E. Rosenberg, S. Bekri, and O. Vizika-Kavvadias, SPE Res. Eval. Eng. 12, 297 (2009).

[6] J.-F. Thovert and P. M. Adler, Phys. Rev. E 83, 056116 (2011).

[7] C. H. Arns, M. A. Knackstedt, M. Val Pinczewski, and W. B. Lindquist, Geophys. Res. Lett. 28, 3361 (2001).

[8] C. Arns, M. Knackstedt, W. Val Pinczewski, and E. Garboczi, J. Geophys. 67, 1396 (2002).

[9] V. V. Mourzenko, O. Galamay, J.-F. Thovert, and P. M. Adler, Phys. Rev. E 56, 3167 (1997).

[10] I. Malinouskaya, Ph.D. thesis, Institut de Physique du Globe de Paris, 2007.

[11] I. Malinouskaya, V. V. Mourzenko, J.-F. Thovert, and P. M. Adler, Phys. Rev. E 77, 066302 (2008).

[12] J. F. Thovert, F. Wary, and P. M. Adler, J. Appl. Phys. 68, 3872 (1990).

[13] P. M. Adler, Porous Media: Geometry and Transports (Butterworth-Heinemann, Stoneham, 1992).

[14] L. Jasinski, Ph.D. thesis, Université Pierre et Marie Curie, Paris, France, 2012.

[15] S. M. Alizadeh, N. Francois, J. Middleton, S. Latham, A. Limaye, T. Senden, and C. H. Arns, International Symposium of the Society of Core Analysts held in Napa Valley, California, USA, 16-19 September (Society of Core Analyst, Houston, 2013).
[16] I. Bogdanov, V. Mourzenko, J.-F. Thovert, and P. M. Adler, Water Resour. Res. 39, 1023 (2003).

[17] R. Lemaître and P. M. Adler, Transport Porous Med. 5, 325 (1990).

[18] R. Peyret and T. D. Taylor, Computational Methods for Fluid Flow, Springer Series in Computational Physics (Springer-Verlag, Berlin, 1985).

[19] R. I. Korsnes, R. Risnes, I. Faldaas, and T. Norland, Tectonophysics 426, 239 (2006).

[20] J.-F. Thovert, F. Yousefian, P. Spanne, C. G. Jacquin, and P. M. Adler, Phys. Rev. E 63, 061307 (2001).

[21] B. Biswal, C. Manwart, and R. Hilfer, Physica A 255, 221 (1998).

[22] A. Papoulis and S. Unnikrishna Pillai, Probability, Random Variables and Stochastic Processes, 4th ed. (McGraw-Hill, New York, 2002).

[23] C. Varloteaux, T. M. Vu, S. Bekri, and P. M. Adler, Phys. Rev. E 87, 023010 (2013).

[24] J.-F. Thovert, J. Sallès, and P. M. Adler, J. Microsc. 170, 65 (1993).

[25] Z. Hashin and S. Shtrikman, J. Mech. Phys. Solids 11, 127 (1963).

[26] G. W. Milton, Phys. Rev. Lett. 46, 542 (1981).

[27] J. G. Berryman, J. Phys. D: Appl. Phys. 18, 585 (1985).

[28] J. Dautriat, Ph.D. thesis, Ecole Polytechnique, Palaiseau, France, 2009.

[29] G. Mavko, T. Mukerji, and J. Dvorkin, The Rock Physics Handbook (Cambridge University Press, Cambridge, 1998).

[30] L. Levien, C. T. Prewitt, and D. J. Weidner, Am. Mineral. 65, 920 (1980).

[31] H. Gercek, Int. J. Rock Mech. Min. Sci. 44, 1 (2007).

[32] J. G. Berryman, J. Geophys. Res. 97, 17409 (1992). 